\title{
Rational terms of UV origin at two loops
}

\author{
Stefano Pozzorini, ${ }^{a}$ Hantian Zhang $^{a}$ and Max F. Zoller ${ }^{b}$ \\ ${ }^{a}$ Physik-Institut, Universität Zürich, CH-8057 Zürich, Switzerland \\ ${ }^{b}$ Paul Scherrer Institut, Forschungsstrasse 111, CH-5232 Villigen PSI, Switzerland \\ E-mail: pozzorin@physik.uzh.ch, hantian.zhang@physik.uzh.ch, \\ max.zoller@psi.ch
}

ABSTRACT: The advent of efficient numerical algorithms for the construction of one-loop amplitudes has played a crucial role in the automation of NLO calculations, and the development of similar algorithms at two loops is a natural strategy for NNLO automation. Within a numerical framework the numerator of loop integrals is usually constructed in four dimensions, and the missing rational terms, which arise from the interplay of the $(D-4)$-dimensional parts of the loop numerator with $1 /(D-4)$ poles in $D$ dimensions, are reconstructed separately. At one loop, such rational terms arise only from UV divergences and can be restored through process-independent local counterterms. In this paper we investigate the behaviour of rational terms of UV origin at two loops. The main result is a general formula that combines the subtraction of UV poles with the reconstruction of the associated rational parts at two loops. This formula has the same structure as the R-operation, and all poles and rational parts are described through a finite set of processindependent local counterterms. We also present a general formula for the calculation of all relevant two-loop rational counterterms in any renormalisable theory based on onescale tadpole integrals. As a first application, we derive the full set of two-loop rational counterterms for QED in the $R_{\xi}$-gauge.

KeYwords: Perturbative QCD, Precision QED

ARXIV EPRINT: 2001.11388 


\section{Contents}

1 Introduction 1

2 Notation and conventions $\quad 4$

2.1 Notation for $D$-dimensional quantities 4

$\begin{array}{lll}2.2 & \text { Reducible and irreducible loop amplitudes } & 6\end{array}$

$\begin{array}{lll}3 & \text { Rational terms at one loop and the tadpole method } & 7\end{array}$

$\begin{array}{lll}3.1 & \text { Rational parts of one-loop diagrams } & 7\end{array}$

$\begin{array}{lll}3.2 & \text { Tadpole decomposition } & 9\end{array}$

$\begin{array}{lll}3.3 & \text { One-loop poles and rational parts in terms of tadpole integrals } & 12\end{array}$

4 One-loop diagrams with $D$-dimensional external momenta 13

$\begin{array}{lll}4.1 & \text { One-loop subdiagram in } D_{\mathrm{n}}=D \text { dimensions } & 14\end{array}$

$\begin{array}{lll}4.2 & \text { One-loop subdiagram in } D_{\mathrm{n}}=4 \text { dimensions } & 15\end{array}$

4.3 Relating renormalised one-loop subdiagrams in $D_{\mathrm{n}}=D$ and $D_{\mathrm{n}}=4 \quad 17$

$\begin{array}{llr}5 & \text { Rational terms at two loops } & \mathbf{1 8}\end{array}$

$\begin{array}{ll}\text { 5.1 Notation for two-loop diagrams and subdiagrams } & 18\end{array}$

$\begin{array}{lll}5.2 & \text { Power counting and structure of UV divergences } & 20\end{array}$

$\begin{array}{lll}5.3 & \text { Structure of UV poles at two loops } & 21\end{array}$

5.4 Structure of rational parts at two loops 22

5.5 Proof and recipe for the calculation of rational terms 24

5.5.1 Diagrams with $X(\Gamma)<0 \quad 24$

$\begin{array}{ll}\text { 5.5.2 Diagrams with } X(\Gamma) \geq 0 & 26\end{array}$

6 Two-loop rational terms in QED 30

$\begin{array}{lll}7 & \text { Summary and conclusions } & 32\end{array}$

A Feynman rules and UV counterterms in QED 33

\section{Introduction}

Higher-order calculations of scattering amplitudes are usually performed in $D=4-2 \varepsilon$ dimensions [1], where the ultraviolet (UV) and infrared (IR) divergences of loop integrals assume the form of $1 / \varepsilon$ poles. ${ }^{1}$ Upon subtraction of all UV and IR singularities, scattering amplitudes become finite in the limit $\varepsilon \rightarrow 0$. Nonetheless they still contain non-vanishing

\footnotetext{
${ }^{1}$ For a review of the different variants of dimensional regularisation see [2].
} 
contributions stemming from the interplay of $1 / \varepsilon$ poles with the $(D-4)$-dimensional parts of loop integrands. For non-trivial processes, depending on the employed technique the explicit calculation of such $(D-4)$-dimensional parts can be technically involved and CPU intensive. For this reason, automated one-loop tools such as OpenLoops [3], Recola [4], HelaC-1Loop [5] and MAdLoop [6] are based on numerical algorithms that construct the numerators of loop integrals in four dimensions, while keeping the denominators in $D$ dimensions. The missing contributions stemming from the $(D-4)$-dimensional parts of loop numerators are easily reconstructed a posteriori through insertions of process-independent rational counterterms [7-10] into tree amplitudes.

More explicitly, let us consider the renormalised amplitude of a one-loop diagram $\gamma$,

$$
\mathbf{R} \overline{\mathcal{A}}_{1, \gamma}=\overline{\mathcal{A}}_{1, \gamma}+\delta Z_{1, \gamma}
$$

where $\overline{\mathcal{A}}_{1, \gamma}$ denotes the unrenormalised amplitude in $D$ dimensions, and $\delta Z_{1, \gamma}$ is the corresponding UV counterterm. In this paper we focus on the contributions that arise when the loop-integrand numerator in $D$ dimensions is split into two parts,

$$
\overline{\mathcal{N}}(\bar{q})=\mathcal{N}(q)+\tilde{\mathcal{N}}(\bar{q})
$$

where $q$ is the loop momentum, and symbols with and without a bar denote, respectively, quantities in $D$ and four dimensions, while $\tilde{\mathcal{N}}(\bar{q})$ is the $(D-4)$-dimensional part of the loop numerator.

At one loop, the interplay of $\tilde{\mathcal{N}}(\bar{q})$ with $1 / \varepsilon$ poles of IR type can generate finite terms at intermediate stages of the calculations, but at the level of full Feynman diagrams such terms cancel $^{2}$ [11]. Thus $\tilde{\mathcal{N}}$-contributions arise only from divergences of UV type. This makes it possible to cast the renormalised amplitude (1.1) in the form

$$
\mathbf{R} \overline{\mathcal{A}}_{1, \gamma}=\mathcal{A}_{1, \gamma}+\delta Z_{1, \gamma}+\delta \mathcal{R}_{1, \gamma}
$$

where $\mathcal{A}_{1, \gamma}$ is the unrenormalised amplitude with numerator $\mathcal{N}(q)$ in four dimensions, $\delta Z_{1, \gamma}$ is the usual $\overline{\mathrm{MS}}$ counterterm, and the extra counterterm $\delta \mathcal{R}_{1, \gamma}$ reconstructs the finite contribution stemming from the part $\tilde{\mathcal{N}}(\bar{q})$ of the numerator. Since $\delta \mathcal{R}_{1, \gamma}$ terms arise only from UV divergences, similarly as the usual UV counterterms they originate only from UV-divergent one-particle irreducible (1PI) subdiagrams, where they take the form of polynomials of the external momenta and internal masses. Thus the insertions of $\delta \mathcal{R}_{1, \gamma}$ counterterms into scattering amplitudes gives rise to rational functions of the kinematic invariants. ${ }^{3}$

The goal of this paper is to extend the reconstruction of $\tilde{\mathcal{N}}$-contributions to two loops, such as to enable two-loop calculations based on numerical tools that build the numerator of Feynman integrals in four dimensions. In our analysis we will focus on two-loop $\tilde{\mathcal{N}}$ contributions of UV origin assuming that IR divergences are either absent, like in off-shell

\footnotetext{
${ }^{2}$ More precisely, $\tilde{\mathcal{N}}$-contributions of IR origin cancel in regularisation schemes where the external degrees of freedom are kept in four dimensions, such as in the 't Hooft-Veltman scheme [1].

${ }^{3}$ In the literature the $\delta \mathcal{R}_{1, \gamma}$ terms in (1.3) are usually denoted as rational terms of type $R_{2}$, and should not be confused with the so-called rational terms of type $R_{1}$. See section 3.1 for more details.
} 
scattering amplitudes, or are subtracted in a way that does not generate rational terms. A systematic analysis of $\tilde{\mathcal{N}}$-contributions of IR origin is deferred to future work.

The reconstruction of $\tilde{\mathcal{N}}$-contributions of UV origin will be carried out at the level of UV-renormalised two-loop amplitudes. In renormalisable theories, the UV renormalisation can be implemented through a recursive procedure that is known as the $\mathbf{R}$-operation [12-15] and amounts to the insertion of local subtraction terms into multi-loop diagrams and their subdiagrams. For the amplitude of a two-loop diagram $\Gamma$, the $\mathbf{R}$-operation has the form

$$
\mathbf{R} \overline{\mathcal{A}}_{2, \Gamma}=\overline{\mathcal{A}}_{2, \Gamma}+\sum_{\gamma} \delta Z_{1, \gamma} \cdot \overline{\mathcal{A}}_{1, \Gamma / \gamma}+\delta Z_{2, \Gamma},
$$

where $\overline{\mathcal{A}}_{2, \Gamma}$ is the unrenormalised two-loop amplitude in $D$ dimensions, and the remaining terms on the r.h.s. correspond to a two-step subtraction. In the first step, the subdivergence of the various one-loop subdiagrams $\gamma$ are subtracted by inserting the counterterms $\delta Z_{1, \gamma}$ into their complementary one-loop diagrams $\Gamma / \gamma$, which are derived from $\Gamma$ by shrinking $\gamma$ to a vertex. In the second step, the remaining local two-loop divergence of $\Gamma$ is subtracted by the local counterterm $\delta Z_{2, \Gamma}$. The identity (1.4) is applicable also when $\Gamma$ is a set of twoloop diagrams. In this case the bookkeeping of $\gamma$ and $\Gamma / \gamma$, which can be single diagrams or sets of diagrams, follows naturally from the case of a single two-loop diagram by using $\mathbf{R}$ as a linear operation. In fact, the $\mathbf{R}$-operation is typically applied at the level of full 1PI vertex functions $\Gamma$ and $\gamma$.

As we will demonstrate, the following generalisation of the $\mathbf{R}$-operation makes it possible to construct renormalised two-loop amplitudes using loop integrands with four-dimensional numerators and rational counterterms for the reconstruction of $\tilde{\mathcal{N}}$-contributions,

$$
\mathbf{R} \overline{\mathcal{A}}_{2, \Gamma}=\mathcal{A}_{2, \Gamma}+\sum_{\gamma}\left(\delta Z_{1, \gamma}+\delta \tilde{Z}_{1, \gamma}+\delta \mathcal{R}_{1, \gamma}\right) \cdot \mathcal{A}_{1, \Gamma / \gamma}+\left(\delta Z_{2, \Gamma}+\delta \mathcal{R}_{2, \Gamma}\right) .
$$

Here the two-loop amplitude $\mathcal{A}_{2, \Gamma}$ and its one-loop parts $\mathcal{A}_{1, \Gamma / \gamma}$ are computed with fourdimensional numerators. The $\overline{\mathrm{MS}}$ counterterms $\delta Z_{2, \Gamma}$ and $\delta Z_{1, \gamma}$ are related to the ones in (1.4) via trivial projection to four dimensions. Quadratically divergent one-loop subdiagrams require additional counterterms $\delta \tilde{Z}_{1, \gamma}$, which subtract extra poles of the form $\tilde{q}^{2} / \varepsilon$, with $\tilde{q}=\bar{q}-q$, that appear as a consequence of the different dimensionality of the loop momenta in the two-loop numerator and denominator. The one-loop UV counterterms are accompanied by related $\delta \mathcal{R}_{1, \gamma}$ counterterms, which reconstruct the $\tilde{\mathcal{N}}$-contributions stemming from subdivergences. Similarly, the two-loop UV counterterms are supplemented by $\delta \mathcal{R}_{2, \gamma}$ counterterms for the reconstruction of the remaining $\tilde{\mathcal{N}}$-contributions, which originate from the local two-loop divergences remaining after the subtraction of all subdivergences.

As we will show, the $\delta \mathcal{R}_{2, \Gamma}$ contributions arise only from superficially divergent 1PI two-loop diagrams and can be reduced to a finite set of process-independent local counterterms. Using a tadpole decomposition technique [16, 17], which is well known from the computation of renormalisation constants and renormalisation group functions, we will derive a general formula for the calculations of the $\delta \mathcal{R}_{2}$ counterterms in any renormalisable theory. Finally, as a first application, we present the full set of two-loop rational counterterms for QED in the $R_{\xi}$-gauge. 
We note that the connection established in this paper between two-loop amplitudes with loop numerators in $D$ and four dimensions bears some similarity to the relations presented in [18] between two-loop QCD vertex functions in dimensional regularisation and in the four-dimensional regularisation/renormalisation (FDR) approach [19]. However, these two studies are based on very different regularisation and renormalisation procedures. In the FDR approach loop integrals are entirely kept in four dimensions, and the divergences are cancelled by means of a set of subtraction rules. In contrast, our approach is based on loop integrals in $D$ dimensions, where only the numerator is restricted to four dimensions, and the contributions stemming from its $(D-4)$-dimensional parts are reconstructed in a way that corresponds exactly to $\overline{\mathrm{MS}}$-renormalised amplitudes in dimensional regularisation. Moreover, we point out that the properties of UV rational terms established in this paper are proven in a fully general way.

The paper is organised as follows. In section 2 we introduce our notation and conventions. In section 3 we review rational terms at one loop, and we introduce the tadpole decomposition method of $[16,17]$, which will be used to calculate rational counterterms and to discuss their general properties. In section 4 we consider one-loop diagrams with $D$-dimensional external loop momenta and the related $\delta \tilde{Z}_{1}$ counterterms. The master formula (1.5) for the reconstruction of rational terms is derived in section 5 , where we also present a general formula for the calculation of the required $\delta \mathcal{R}_{2}$ counterterms. Explicit results for such counterterms in QED can be found in section 6, and the $\overline{\mathrm{MS}}$ counterterms for $\mathrm{QED}$ in the $R_{\xi}$ gauge are listed in appendix $\mathrm{A}$.

\section{Notation and conventions}

In this section we introduce our conventions for the treatment of dimensionally regularised scattering amplitudes and for their decomposition into irreducible loop subdiagrams and tree subdiagrams.

\subsection{Notation for $D$-dimensional quantities}

For the regularisation of UV divergences in this paper we use the 't Hooft-Veltman scheme [1], where external states are four-dimensional, while loop momenta as well as the metric tensors and Dirac matrices inside the loops live in

$$
D=4-2 \varepsilon
$$

dimensions. For the analysis of rational terms we use an additional parameter $D_{\mathrm{n}}$, which denotes the dimensionality of loop numerators and can take the values

$$
D_{\mathrm{n}}= \begin{cases}D & \text { for calculations in } D \text { dimensions } \\ 4 & \text { for calculations with four-dimensional loop numerator }\end{cases}
$$

Amplitudes with loop numerators in four dimensions and loop denominators in $D$ dimensions will be referred to as $D_{\mathrm{n}}=4$ dimensional amplitudes. 
In $D_{\mathrm{n}}=D$ dimensions, all relevant ingredients of loop numerators will be decomposed into four-dimensional parts and $(D-4)$-dimensional remnants. Contractions of Lorentz vectors in $D$ dimensions are decomposed as

$$
A_{\bar{\mu}} B^{\bar{\mu}}=A_{\mu} B^{\mu}+A_{\tilde{\mu}} B^{\tilde{\mu}},
$$

where the indices $\bar{\mu}$ range over all components of the $D$-dimensional vectors, the indices $\mu$ are restricted to four dimensions, and the indices $\tilde{\mu}$ are associated with the $(D-4)$ dimensional remnant. In general, to distinguish $D$ and $(D-4)$-dimensional quantities from their four-dimensional counterparts we use symbols carrying a bar and a tilde, respectively. For the $D$-dimensional loop momentum we write

$$
\bar{q}=q+\tilde{q},
$$

where

$$
q^{\mu}=\bar{q}^{\mu}, \quad \tilde{q}^{\tilde{\mu}}=\bar{q}^{\tilde{\mu}},
$$

and

$$
\bar{q}^{2}=q^{2}+\tilde{q}^{2}
$$

For the integration measure in loop-momentum space we use the shorthand

$$
\int \mathrm{d} \bar{q}=\mu^{2 \varepsilon} \int \frac{\mathrm{d}^{D} \bar{q}}{(2 \pi)^{D}}
$$

where $\mu$ is the scale of dimensional regularisation and will be identified with the renormalisation scale.

Given that $q^{\tilde{\mu}}=\tilde{q}^{\mu}=0$, for the Lorentz indices of $q$ and $\tilde{q}$ we often use a sloppy notation where we identify $q^{\bar{\mu}} \equiv q^{\mu}$ and $\tilde{q}^{\bar{\mu}} \equiv \tilde{q}^{\tilde{\mu}}$. Thus (2.4) will be typically written as

$$
\bar{q}^{\bar{\mu}}=q^{\mu}+\tilde{q}^{\tilde{\mu}} .
$$

This leads to contractions of objects that carry different kinds of indices and have to be understood as follows,

$$
A_{\bar{\mu}} B^{\mu}=A_{\mu} B^{\mu}, \quad A_{\bar{\mu}} B^{\tilde{\mu}}=A_{\tilde{\mu}} B^{\tilde{\mu}}, \quad A_{\mu} B^{\tilde{\mu}}=0 .
$$

A similar notation is used also for the decomposition of Dirac matrices and the metric tensor,

$$
\begin{aligned}
\bar{\gamma}^{\mu} & =\gamma^{\mu}+\tilde{\gamma}^{\tilde{\mu}}, \\
\bar{g}^{\bar{\mu} \bar{\nu}} & =g^{\mu \nu}+\tilde{g}^{\tilde{\mu} \tilde{\nu}} .
\end{aligned}
$$

Metric tensors with indices of different type should be understood as

$$
\bar{g}^{\mu \bar{\nu}}=g^{\mu \bar{\nu}}=g^{\mu \nu} .
$$




\subsection{Reducible and irreducible loop amplitudes}

Our analysis of rational terms of UV origin will be carried out at the level of UV-renormalised amplitudes. Before renormalisation, the amplitude of a one-loop diagram $\gamma$ has the general form

$$
\overline{\mathcal{M}}_{1, \gamma}=\underbrace{w_{w_{N-1}}}_{w_{w_{2}}}=\overline{\mathcal{A}}_{1, \gamma}^{\sigma_{1} \ldots \sigma_{N}} \prod_{i=1}^{N}\left[w_{i}\right]_{\sigma_{i}},
$$

where $\overline{\mathcal{A}}_{1, \gamma}$ corresponds to the amplitude of the 1PI amputated one-loop subdiagram of $\gamma$, which is connected to the external lines through the factorised subtrees $w_{i}$, depicted as blue bubbles. We denote as subtree a tree subdiagram that connects an internal vertex to a set of external lines. Since external subtrees are free from UV singularities, only the 1PI subdiagram needs to be renormalised, i.e.

$$
\mathbf{R} \overline{\mathcal{M}}_{1, \gamma}=\left(\mathbf{R} \overline{\mathcal{A}}_{1, \gamma}^{\sigma_{1} \ldots \sigma_{N}}\right) \prod_{i=1}^{N}\left[w_{i}\right]_{\sigma_{i}} .
$$

Two-loop diagrams can be classified into two types depending on whether the topology that results from the amputation of all external subtrees is irreducible or still reducible. The amplitude of a two-loop diagram $\Gamma$ of the first type has the form

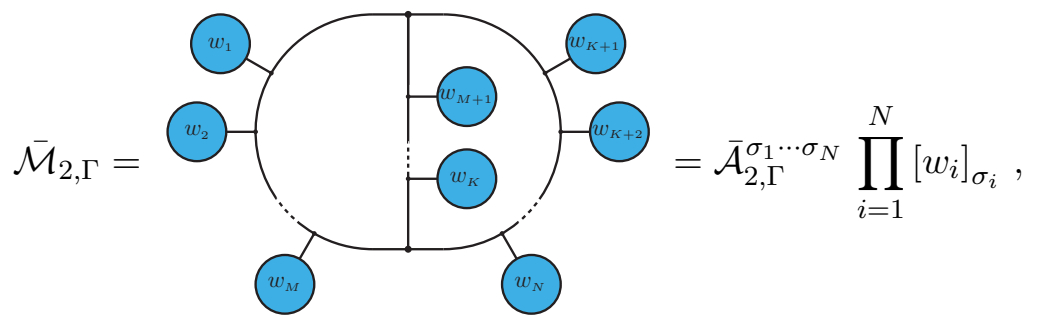

where $\overline{\mathcal{A}}_{2, \Gamma}$ corresponds to the amplitude of the 1PI amputated two-loop diagram that is left after factorisation of all external subtrees $w_{i}$. Similarly as in the one-loop case, the R-operation acts only on the 1PI part,

$$
\mathbf{R} \overline{\mathcal{M}}_{2, \Gamma}=\left(\mathbf{R} \overline{\mathcal{A}}_{2, \Gamma}^{\sigma_{1} \cdots \sigma_{N}}\right) \prod_{i=1}^{N}\left[w_{i}\right]_{\sigma_{i}} .
$$

The general form of the amplitude of a two-loop diagram $\Gamma_{\text {red }}$ of the second type is

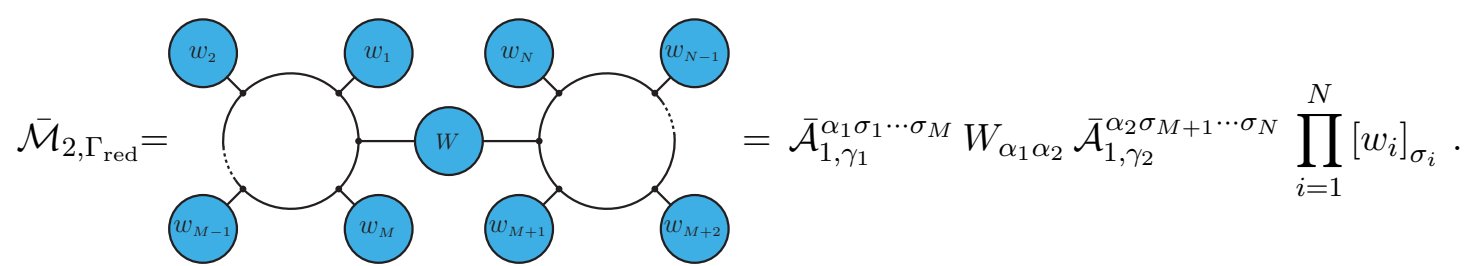


Here, the factorisation of all external subtrees $w_{i}$ leads to two separate 1PI amputated one-loop amplitudes, $\overline{\mathcal{A}}_{1, \gamma_{1}}$ and $\overline{\mathcal{A}}_{1, \gamma_{2}}$, that are connected to each other through a tree structure $W$. Also in this case the $\mathbf{R}$-operation acts only on the 1PI building blocks,

$$
\mathbf{R} \overline{\mathcal{M}}_{2, \Gamma_{\mathrm{red}}}=\left(\mathbf{R} \overline{\mathcal{A}}_{1, \gamma}^{\alpha_{1} \sigma_{1} \cdots \sigma_{M}}\right) W_{\alpha_{1} \alpha_{2}}\left(\mathbf{R} \overline{\mathcal{A}}_{1, \gamma}^{\alpha_{2} \sigma_{M+1} \cdots \sigma_{N}}\right) \prod_{i=1}^{N}\left[w_{i}\right]_{\sigma_{i}} .
$$

In this paper we will consider $\overline{\mathrm{MS}}$ renormalised amplitudes in the 't Hooft-Veltman scheme, where all tree structures $w_{i}$ and $W$ in $(2.12)-(2.17)$ are in four dimensions. Thus the external momenta and external indices of the 1PI amplitudes $\overline{\mathcal{A}}_{1, \gamma}$ and $\overline{\mathcal{A}}_{2, \Gamma}$ are handled as four-dimensional quantities. Since they are free from $(D-4)$-dimensional parts, in the 't Hooft-Veltman scheme tree structures do not generate any rational term. ${ }^{4}$ Thus rational terms can be determined at the level of 1PI subdiagrams and directly extended to full amplitudes through (2.13) and (2.15)-(2.17).

\section{Rational terms at one loop and the tadpole method}

This section deals with the structure of rational terms at one loop and their connection with UV poles. In this context we introduce a general technique that makes it possible to reduce rational terms of UV origin to tadpole integrals.

\subsection{Rational parts of one-loop diagrams}

Let us consider the amplitude of a one-particle irreducible one-loop diagram $\gamma$,

$$
\overline{\mathcal{A}}_{1, \gamma}=\int \mathrm{d} \bar{q}_{1} \frac{\overline{\mathcal{N}}\left(\bar{q}_{1}\right)}{D_{0}\left(\bar{q}_{1}\right) \cdots D_{N-1}\left(\bar{q}_{1}\right)},
$$

with denominators

$$
D_{j}\left(\bar{q}_{1}\right)=\left(\bar{q}_{1}+p_{j}\right)^{2}-m_{j}^{2}
$$

where

$$
p_{j}^{\mu}=\sum_{i=1}^{j} k_{i}^{\mu}
$$

and $k_{1}, \ldots, k_{N}$ are the $N$ external momenta flowing into the loop. Momentum conservation implies $\sum_{i=1}^{N} k_{i}=0$. Colour structures and all Lorentz or Dirac indices associated with the amputated external legs that enter the loop are implicitly understood. Such indices as well as all external momenta are treated as four-dimensional quantities as discussed in section 2.2 .

In $D_{\mathrm{n}}=D$ dimensions, the numerator $\overline{\mathcal{N}}\left(\bar{q}_{1}\right)$ can be split into

$$
\overline{\mathcal{N}}\left(\bar{q}_{1}\right)=\mathcal{N}\left(q_{1}\right)+\tilde{\mathcal{N}}\left(\bar{q}_{1}\right),
$$

\footnotetext{
${ }^{4}$ As far as rational terms of UV origin are concerned, the $(D-4)$-parts of tree structures, which appear in alternative schemes such as conventional dimensional regularisation, are irrelevant since they factorise with respect to the UV-renormalised 1PI subdiagrams.
} 
where

$$
\mathcal{N}\left(q_{1}\right)=\left.\overline{\mathcal{N}}\left(\bar{q}_{1}\right)\right|_{\bar{g} \rightarrow g, \bar{\gamma} \rightarrow \gamma, \bar{q}_{1} \rightarrow q_{1}}
$$

is the four-dimensional part, obtained by projecting the metric tensor, Dirac matrices and the loop momentum to four dimensions. By construction, the remnant $\tilde{\mathcal{N}}\left(\bar{q}_{1}\right)$ vanishes in $D_{\mathrm{n}}=4$ dimensions. More precisely,

$$
\tilde{\mathcal{N}}\left(\bar{q}_{1}\right)=\mathcal{O}\left(\varepsilon, \tilde{q}_{1}\right)
$$

in $D_{\mathrm{n}}=4-2 \varepsilon$ dimensions. Thus we will refer to $\tilde{\mathcal{N}}$ as the $(D-4)$-dimensional part of the numerator.

At the level of the one-loop amplitude the splitting (3.4) results into

$$
\overline{\mathcal{A}}_{1, \gamma}=\mathcal{A}_{1, \gamma}+\delta \mathcal{R}_{1, \gamma},
$$

where

$$
\mathcal{A}_{1}=\int \mathrm{d} \bar{q}_{1} \frac{\mathcal{N}\left(q_{1}\right)}{D_{0}\left(\bar{q}_{1}\right) \cdots D_{N-1}\left(\bar{q}_{1}\right)}
$$

can be computed with tools that handle the numerator in $D_{\mathrm{n}}=4$ dimensions while retaining the full $D$-dependence of the loop momentum in the denominator. The remnant part,

$$
\delta \mathcal{R}_{1, \gamma}=\int \mathrm{d} \bar{q}_{1} \frac{\tilde{\mathcal{N}}\left(\bar{q}_{1}\right)}{D_{0}\left(\bar{q}_{1}\right) \cdots D_{N-1}\left(\bar{q}_{1}\right)},
$$

will be referred to as $\tilde{\mathcal{N}}$-contribution. Here the only relevant terms are the $\mathcal{O}\left(\varepsilon^{0}\right)$ contributions that originate from the interplay of the $(D-4)$-dimensional part of the numerator with $1 / \varepsilon$ poles. At one loop such $\tilde{\mathcal{N}}$-contributions originate only from poles of UV type [11], and similarly as for UV poles they arise only from UV divergent 1PI functions, where they take the form of simple polynomials in the external momenta and internal masses. For this reason, $\tilde{\mathcal{N}}$-contributions can be reconstructed through a finite set of process-independent counterterms [7-10]. Their insertion into tree amplitudes gives rise to rational functions of the kinematic invariants.

In the literature, the one-loop terms that arise from the $(D-4)$-dimensional part of the loop denominators in (3.8) and from $\tilde{\mathcal{N}}$ are denoted, respectively, rational terms of type $R_{1}$ and $R_{2}$. The rational terms of type $R_{1}$ emerge from the reduction of tensor integrals to scalar integrals and can be handled with numerical algorithms in four dimensions (see e.g. [20, 21]). However, they can not be reduced to a finite set of counterterms. In this paper we will focus on the rational terms that originate from $\tilde{\mathcal{N}}$ at one and two loops. Since we consider a single type of rational terms, for convenience we will use the symbols $\delta \mathcal{R}_{L}$, where $L=1,2, \ldots$ indicates the loop order and not the kind of rational term. We will refer to such contributions as $\tilde{\mathcal{N}}$ rational terms or simply rational terms.

We note that the relation (3.7) may be regarded as a regularisation-scheme transformations that connects the amplitude $\overline{\mathcal{A}}_{1, \gamma}$ in the 't Hooft-Veltman scheme to its counterpart $\mathcal{A}_{1, \gamma}$ in a pseudo-regularisation scheme corresponding to the prescription (3.5). However, we point out that the four-dimensional projection (3.5) breaks gauge invariance and cannot 
be regarded as a consistent regularisation prescription. Only the combination of the two terms on the r.h.s. of (3.7) should be regarded as a consistently regularised amplitude, and - by construction - this combination is equivalent to the 't Hooft-Veltman scheme. We also note that the prescription (3.5) should not be confused with the four-dimensional helicity scheme $(\mathrm{FDH})[22,23]$, where the $(D-4)$-dimensional part of the loop momentum is retained throughout. ${ }^{5}$

\subsection{Tadpole decomposition}

In this section we discuss a general method [16, 17, 25] that makes it possible to cast the UV divergent parts of loop integrals - which are at the origin of rational terms - in the form of tadpole integrals. This method is first introduced for one-loop integrals, while its application to two-loop integrals is discussed in section 5.5.

For the analysis of UV divergences it is convenient to express one-loop amplitudes in terms of tensor integrals,

$$
T_{N}^{\bar{\mu}_{1} \cdots \bar{\mu}_{r}}=\int \mathrm{d} \bar{q}_{1} \frac{\bar{q}_{1}^{\bar{\mu}_{1}} \cdots \bar{q}_{1}^{\bar{\mu}_{r}}}{D_{0}\left(\bar{q}_{1}\right) \cdots D_{N-1}\left(\bar{q}_{1}\right)} .
$$

In the case of the one-loop amplitude (3.1) we have

$$
\overline{\mathcal{A}}_{1, \gamma}=\sum_{r=0}^{R} \overline{\mathcal{N}}_{\bar{\mu}_{1} \cdots \bar{\mu}_{r}} T_{N}^{\bar{\mu}_{1} \cdots \bar{\mu}_{r}},
$$

where the coefficients $\overline{\mathcal{N}}_{\bar{\mu}_{1} \cdots \bar{\mu}_{r}}$ depend on the external momenta and helicities, and are related to the loop numerator via

$$
\overline{\mathcal{N}}\left(\bar{q}_{1}\right)=\sum_{r=0}^{R} \overline{\mathcal{N}}_{\bar{\mu}_{1} \cdots \bar{\mu}_{r}} \bar{q}_{1}^{\bar{\mu}_{1}} \cdots \bar{q}_{1}^{\bar{\mu}_{r}} .
$$

The loop integrals (3.10) give rise to UV singularities if their integrands scale like $q^{X}$ with $X \geq 0$ at $q \rightarrow \infty$. The power $X$ is referred to as superficial degree of divergence and can be determined via naive power counting in $q$. For the tensor integrals (3.10) it is given by

$$
X=4+r-2 N,
$$

and the tensor rank $r$ fulfils $r \leq R \leq N$ in renormalisable theories.

In order to isolate UV poles, it is convenient to separate the loop denominators into leading and subleading UV parts according to

$$
D_{k}\left(\bar{q}_{1}\right)=\left(\bar{q}_{1}^{2}-M^{2}\right)-\Delta_{k}\left(\bar{q}_{1}\right)
$$

with

$$
\Delta_{k}\left(\bar{q}_{1}\right)=-p_{k}^{2}-2 \bar{q}_{1} \cdot p_{k}+m_{k}^{2}-M^{2},
$$

\footnotetext{
${ }^{5}$ At one loop, scattering amplitudes in the FDH scheme can be reconstructed in terms of loop integrals with four-dimensional numerators using the FDF approach [24].
} 
where $M$ is an auxiliary mass scale. ${ }^{6}$ The dominant UV contribution of $\mathcal{O}\left(q_{1}^{2}\right)$ is captured by the term $\left(\bar{q}_{1}^{2}-M^{2}\right)$, which corresponds to the form of a massive tadpole propagator, while $\Delta_{k}\left(\bar{q}_{1}\right)$ is a subleading contribution of $\mathcal{O}\left(q_{1}^{1}\right)$. Note that for one-loop amplitudes with four-dimensional external momenta the $(D-4)$-dimensional part of the loop momentum does not contribute to 3.15 , i.e. $\Delta_{k}\left(\bar{q}_{1}\right)=\Delta_{k}\left(q_{1}\right)$. In contrast, the external momenta of a one-loop subdiagram that is embedded in a two-loop diagram can depend on the second loop momentum $\bar{q}_{2}$, giving rise to $D$-dimensional terms of the form $-\bar{q}_{2}^{2} \pm 2 \bar{q}_{1} \cdot \bar{q}_{2}$ in $\Delta_{k}\left(\bar{q}_{1}\right)$.

Inverting the l.h.s. and the r.h.s. of (3.14) and using partial fractioning leads to the tadpole decomposition formula $[16,17]$

$$
\frac{1}{D_{k}\left(\bar{q}_{1}\right)}=\frac{1}{\bar{q}_{1}^{2}-M^{2}}+\frac{\Delta_{k}\left(\bar{q}_{1}\right)}{\bar{q}_{1}^{2}-M^{2}} \frac{1}{D_{k}\left(\bar{q}_{1}\right)}
$$

which separates a generic scalar propagator into a leading tadpole contribution of order $1 / q_{1}^{2}$ and a subleading remnant consisting of the original propagator times an extra suppression factor of order ${ }^{7} 1 / q_{1}$. The identity (3.16) holds exactly, and its recursive application makes it possible to generate a systematic expansion of the propagators in the limit $1 / q_{1} \rightarrow 0$. More explicitly, applying (3.16) $X+1$ times yields

$$
\frac{1}{D_{k}\left(\bar{q}_{1}\right)}=\sum_{\sigma=0}^{X} \frac{\left[\Delta_{k}\left(\bar{q}_{1}\right)\right]^{\sigma}}{\left(\bar{q}_{1}^{2}-M^{2}\right)^{\sigma+1}}+\frac{\left[\Delta_{k}\left(\bar{q}_{1}\right)\right]^{X+1}}{\left(\bar{q}_{1}^{2}-M^{2}\right)^{X+1}} \frac{1}{D_{k}\left(\bar{q}_{1}\right)},
$$

where the sum on the r.h.s. consists of pure tadpole terms of order $1 / q_{1}^{2}, \ldots, 1 / q_{1}^{X+2}$ and corresponds to the first $X+1$ terms of the Taylor expansion of

$$
\frac{1}{D_{k}\left(\bar{q}_{1}\right)}=\frac{1}{\bar{q}_{1}^{2}-M^{2}}\left[1+\frac{\Delta_{k}\left(\bar{q}_{1}\right)}{\bar{q}_{1}^{2}-M^{2}}\right]^{-1}
$$

in the expansion parameter $\Delta_{k}\left(\bar{q}_{1}\right) /\left(\bar{q}_{1}^{2}-M^{2}\right)$. The exact remnant of such a truncated expansion, i.e. all missing contributions of order $1 / q_{1}^{X+3}$ and higher, is captured by the term involving the original propagator on the r.h.s. of (3.17).

In order to render (3.17) and similar decomposition formulas more compact, we introduce two operators that generate the truncated expansion in $\Delta_{k}\left(\bar{q}_{1}\right) /\left(\bar{q}_{1}^{2}-M^{2}\right)$ and its remnant, respectively. Specifically, for the two terms on the r.h.s. of (3.17) we write ${ }^{8}$

$$
\mathbf{S}_{X}^{(1)} \frac{1}{D_{k}\left(\bar{q}_{1}\right)}=\sum_{\sigma=0}^{X} \frac{\left[\Delta_{k}\left(\bar{q}_{1}\right)\right]^{\sigma}}{\left(\bar{q}_{1}^{2}-M^{2}\right)^{\sigma+1}}, \quad \mathbf{F}_{X}^{(1)} \frac{1}{D_{k}\left(\bar{q}_{1}\right)}=\frac{\left[\Delta_{k}\left(\bar{q}_{1}\right)\right]^{X+1}}{\left(\bar{q}_{i}^{2}-M^{2}\right)^{X+1}} \frac{1}{D_{k}\left(\bar{q}_{1}\right)} .
$$

More generally, at the level of the full one-loop integrand the above operators are defined as an exact decomposition,

$$
\mathbf{S}_{X}^{(1)}+\mathbf{F}_{X}^{(1)}=1
$$

\footnotetext{
${ }^{6}$ Note that only the squared scale $M^{2}$ appears.

${ }^{7}$ For propagators with $p_{k}=0$ the extra suppression factor is of order $1 / q_{1}^{2}$.

${ }^{8}$ The superscript in $\mathbf{S}_{X}^{(1)}$ and $\mathbf{F}_{X}^{(1)}$ refers to the chain of $q_{1}$-dependent propagator denominators on which the operator acts.
} 
and they act only on the $q_{1}$-dependent chain of loop denominators, i.e.

$$
\mathbf{S}_{X}^{(1)} \frac{\overline{\mathcal{N}}\left(\bar{q}_{1}\right)}{D_{0}\left(\bar{q}_{1}\right) \cdots D_{N-1}\left(\bar{q}_{1}\right)}=\overline{\mathcal{N}}\left(\bar{q}_{1}\right) \mathbf{S}_{X}^{(1)} \frac{1}{D_{0}\left(\bar{q}_{1}\right) \cdots D_{N-1}\left(\bar{q}_{1}\right)},
$$

and similarly for $\mathbf{F}_{X}^{(1)}=1-\mathbf{S}_{X}^{(1)}$. These two operators define a tadpole decomposition up to order $X$ of the entire chain of propagators, where $\mathbf{S}_{X}^{(1)}$ collects all pure tadpole terms with denominators $\left(\bar{q}_{1}^{2}-M^{2}\right)^{N+\sigma}$ and $\sigma \in[0, X]$, while $\mathbf{F}_{X}^{(1)}$ corresponds to the remnant. More precisely, $\mathbf{S}_{X}^{(1)}$ amounts to a Taylor expansion of the full chain of propagators up to total order $X$ in the various $\Delta_{k}\left(\bar{q}_{1}\right) /\left(\bar{q}_{1}^{2}-M^{2}\right)$, i.e.

$$
\mathbf{S}_{X}^{(1)} \frac{1}{D_{0}\left(\bar{q}_{1}\right) \cdots D_{N-1}\left(\bar{q}_{1}\right)}=\sum_{\sigma=0}^{X} \frac{\Delta^{(\sigma)}\left(\bar{q}_{1}\right)}{\left(\bar{q}_{1}^{2}-M^{2}\right)^{N+\sigma}},
$$

with

$$
\Delta^{(\sigma)}\left(\bar{q}_{1}\right)=\left.\sum_{\sigma_{0}=0}^{\sigma} \ldots \sum_{\sigma_{N-1}=0}^{\sigma} \prod_{k=0}^{N-1}\left[\Delta_{k}\left(\bar{q}_{1}\right)\right]^{\sigma_{k}}\right|_{\sigma_{0}+\cdots+\sigma_{N-1}=\sigma} .
$$

Thus $\mathbf{S}_{X}^{(1)}$ turns the original integrals into a combination of massive tadpole integrals that include all terms from order $1 / q_{1}^{2 N}$ to order $1 / q_{1}^{(2 N+X)}$. The numerators $\Delta^{(\sigma)}\left(\bar{q}_{1}\right)$ of such tadpole integrals are polynomials of degree $\sigma$ in $\bar{q}_{1} \cdot p_{k}$ and in the squared mass scales $\left\{p_{k}^{2}\right\},\left\{m_{k}^{2}\right\}$ and $M^{2}$. By construction, the remainder part associated with $\mathbf{F}_{X}^{(1)}$ involves only terms where the original degree of UV singularity is reduced by $X+1$ or more, i.e. formally

$$
\mathbf{F}_{X}^{(1)} \leq \mathcal{O}\left(\frac{1}{q^{X+1}}\right)
$$

Note also that the $\mathbf{F}_{X}^{(1)}$ remainder embodies all possible IR poles of the original integral since the $\mathbf{S}_{X}^{(1)}$ operator converts the denominators of all massless propagators into massive ones.

In practice, the expansion (3.22)-(3.23) can be generated by applying the decomposition (3.16) in a recursive way until terms with denominators of the form

$$
\left(\bar{q}_{1}^{2}-M^{2}\right)^{p} D_{j_{1}}\left(\bar{q}_{1}\right) \cdots D_{j_{q}}\left(\bar{q}_{1}\right)
$$

with $p+q>N+X$ are encountered, and attributing such terms to $\mathbf{F}_{X}^{(1)}$. Note that, according to the above definition of the tadpole expansion, the $\mathbf{S}_{X}^{(1)}$ operator captures all terms up to relative order $1 / q_{1}^{X}$ but retains also unnecessary terms of higher order in $1 / q_{1}$. This is due to the fact that terms of $\mathcal{O}\left(q_{1}^{1}\right)$ and $\mathcal{O}\left(q_{1}^{0}\right)$ in (3.15) are treated on the same footing. Possible optimisations based on power counting in $1 / q_{1}$ and other tricks are briefly discussed in section 5.5.

For integrals with UV degree of divergence $X$, contributions that are suppressed by a relative factor $1 / q^{X+1}$ do not contribute to the divergence. Thus, using (3.21)-(3.22) we can express the pole part of the tensor integral (3.10) in terms of tadpole integrals with one auxiliary mass scale $M$,

$$
\mathbf{K} T_{N}^{\bar{\mu}_{1} \cdots \bar{\mu}_{r}}=\mathbf{K} \mathbf{S}_{X}^{(1)} T_{N}^{\bar{\mu}_{1} \cdots \bar{\mu}_{r}}=\sum_{\sigma=0}^{X} \mathbf{K} \int \mathrm{d} \bar{q}_{1} \frac{\bar{q}_{1}^{\bar{\mu}_{1}} \cdots \bar{q}_{1}^{\bar{\mu}_{r}} \Delta^{(\sigma)}\left(\bar{q}_{1}\right)}{\left(\bar{q}_{1}^{2}-M^{2}\right)^{N+\sigma}}
$$


Here and in the following $\mathbf{K}$ should be understood as a linear operator that isolates the pole part of an integral and discards the finite remnant. More precisely, let us consider the typical form of the Laurent series that result from $L$-loop integrals,

$$
f_{L}(\varepsilon)=S^{L \varepsilon} \sum_{k=1}^{L} \frac{f_{\overline{\mathrm{MS}}, k}}{\varepsilon^{k}}+f_{\overline{\mathrm{MS}}, 0}+\mathcal{O}(\varepsilon)=\sum_{k=1}^{L} \frac{f_{\mathrm{MS}, k}}{\varepsilon^{k}}+f_{\mathrm{MS}, 0}+\mathcal{O}(\varepsilon),
$$

where

$$
S^{\varepsilon}=(4 \pi)^{\varepsilon} \Gamma(1+\varepsilon)=1+\varepsilon\left[\ln (4 \pi)-\gamma_{\mathrm{E}}\right]+\ldots
$$

is the well-known universal factor associated with each loop-momentum integration. In the MS scheme the $\mathbf{K}$ operator is defined as

$$
\mathbf{K} f_{L}(\varepsilon) \stackrel{\mathrm{MS}}{=} \sum_{k=1}^{L} \frac{f_{\mathrm{MS}, k}}{\varepsilon^{k}}
$$

while in the $\overline{\mathrm{MS}}$ scheme it should be understood as

$$
\mathbf{K} f_{L}(\varepsilon) \stackrel{\overline{\mathrm{MS}}}{=} S^{L \varepsilon} \sum_{k=1}^{L} \frac{f_{\overline{\mathrm{MS}}, k}}{\varepsilon^{k}}
$$

Since the full tadpole decomposition (3.20) is independent of $M$, and the truncated $\mathbf{F}_{X}^{(1)}$ part does not contribute to the divergence, the $M$-dependence of the tadpole integrals cancels on the r.h.s. of (3.26). Moreover, the general form of (3.22)-(3.23) implies that the pole residues are homogenous polynomials of degree $X$ in the external momenta and internal masses.

The above tadpole decomposition can be used also at two loops (and beyond). To this end, as detailed in section 5.5, two-loop integrals are split into the three chains of propagators that depend on the loop momenta $q_{1}, q_{2}$ and $q_{3}=-q_{1}-q_{2}$, and two-loop divergencies are extracted by means of three separate tadpole decompositions with operators $\mathbf{S}_{X_{i}}^{(i)}=1-\mathbf{F}_{X_{i}}^{(i)}$ that act on the particular chain of $q_{i}$-dependent denominators, for $i=1,2,3$, and are otherwise defined as in (3.20)-(3.24).

\subsection{One-loop poles and rational parts in terms of tadpole integrals}

In order to highlight the connection between UV poles and rational $\tilde{\mathcal{N}}$-contributions, we introduce an operator $\overline{\mathbf{K}}$ that extracts the full contribution of UV poles at the level of one-loop amplitudes in $D_{\mathrm{n}}=D$ dimensions. For the generic one-loop amplitude (3.1), using the tensor decomposition (3.11), we define the $\overline{\mathbf{K}}$ operator as

$$
\overline{\mathbf{K}} \overline{\mathcal{A}}_{1, \gamma}=\overline{\mathbf{K}} \sum_{r=0}^{R} \overline{\mathcal{N}}_{\bar{\mu}_{1} \cdots \bar{\mu}_{r}} T_{N}^{\bar{\mu}_{1} \cdots \bar{\mu}_{r}}=\sum_{r=0}^{R} \overline{\mathcal{N}}_{\bar{\mu}_{1} \cdots \bar{\mu}_{r}} \mathbf{K} T_{N}^{\bar{\mu}_{1} \cdots \bar{\mu}_{r}}
$$

and we split it into two pieces,

$$
\overline{\mathbf{K}} \overline{\mathcal{A}}_{1, \gamma}=\mathbf{K} \overline{\mathcal{A}}_{1, \gamma}+\tilde{\mathbf{K}} \mathcal{A}_{1, \gamma},
$$


which result, respectively, from the interplay of the UV poles $\mathbf{K} T_{N}^{\bar{\mu}_{1} \cdots \bar{\mu}_{r}}$ with the fourdimensional and $(D-4)$-dimensional parts of $\overline{\mathcal{N}}_{\bar{\mu}_{1} \cdots \bar{\mu}_{r}}$. The former yields the UV singularity

$$
\mathbf{K} \overline{\mathcal{A}}_{1, \gamma}=\sum_{r=0}^{R} \mathcal{N}_{\mu_{1} \cdots \mu_{r}} \mathbf{K} T_{N}^{\mu_{1} \cdots \mu_{r}}=-\delta Z_{1, \gamma},
$$

where $\delta Z_{1, \gamma}$ is the UV counterterm for the amplitude at hand, while the $(D-4)$-dimensional part of the numerator gives rise to the $\tilde{\mathcal{N}}$-contribution ${ }^{9}$

$$
\tilde{\mathbf{K}} \mathcal{A}_{1, \gamma}=\sum_{r=0}^{R}\left[\overline{\mathcal{N}}_{\bar{\mu}_{1} \cdots \bar{\mu}_{r}}-\mathcal{N}_{\mu_{1} \cdots \mu_{r}}\right] \mathbf{K} T_{N}^{\bar{\mu}_{1} \cdots \bar{\mu}_{r}}=\delta \mathcal{R}_{1, \gamma} .
$$

Note that the difference within square brackets can be regarded as the combination of two kinds of $(D-4)$-dimensional terms: a contribution $\overline{\mathcal{N}}_{\bar{\mu}_{1} \cdots \bar{\mu}_{r}}-\overline{\mathcal{N}}_{\mu_{1} \cdots \mu_{r}}$ that originates from the $(D-4)$-dimensional components of the loop momentum in the tensor integrals (3.10), and a second contribution $\overline{\mathcal{N}}_{\mu_{1} \cdots \mu_{r}}-\mathcal{N}_{\mu_{1} \cdots \mu_{r}}$ that corresponds to the remaining $(D-4)$ dimensional part of the loop numerator.

In renormalisable theories UV singularities at one loop arise only from diagrams with $N \leq 4$ loop propagators. Thus, UV poles and $\tilde{\mathcal{N}}$-contributions can be derived once and for all at the level of the relevant $1 \mathrm{PI}$ vertex functions and encoded in a finite set of $\delta Z_{1, \gamma}$ and $\delta \mathcal{R}_{1, \gamma}$ counterterms. The identities (3.33)-(3.34) can be regarded as the master formulas for the derivation of such counterterms. To this end, the poles of tensor integrals can be computed in terms of tadpole integrals using (3.26). As discussed above, the residues of such poles are $M$-independent polynomials of the external momenta $\left\{p_{k}\right\}$ and internal masses $\left\{m_{k}\right\}$. As a consequence, at the level of $1 \mathrm{PI}$ vertex functions, $\delta Z_{1, \gamma}$ and $\delta \mathcal{R}_{1, \gamma}$ are local counterterms. More precisely, they take the form of homogeneous polynomials of degree $X$ in the external momenta $\left\{p_{k}\right\}$ and internal masses $\left\{m_{k}\right\}$, while their insertion at the level of full scattering amplitudes yields rational functions of the kinematic invariants [7-10].

In section 5, using a similar strategy based on tadpole decompositions and power counting, we demonstrate that also two-loop $\tilde{\mathcal{N}}$-contributions of UV origin can be reconstructed by means of a finite set of local counterterms.

\section{One-loop diagrams with $D$-dimensional external momenta}

As a preparation for the discussion of $\tilde{\mathcal{N}}$-contributions at two loops, in this section we extend the analysis of one-loop UV poles and $\tilde{\mathcal{N}}$-terms to the case of one-loop subdiagrams of two-loop diagrams. Specifically, as depicted in figure 1, we consider one-loop subdiagrams with internal loop momentum $\bar{q}_{1}$ and two external lines that depend on the loop momentum $\bar{q}_{2}$ and are going to be embedded in a two-loop diagram. In the following $\bar{q}_{2}$ is kept fixed, and we investigate the role of its $(D-4)$-dimensional part $\tilde{q}_{2}$. In particular, we show that non-logarithmic UV subdivergences can give rise to non-trivial contributions of the form $\tilde{q}_{2}^{2} / \varepsilon$.

\footnotetext{
${ }^{9}$ On the l.h.s. of (3.34) we write $\mathcal{A}_{1, \gamma}$ without bar since, a posteriori, $\tilde{\mathbf{K}} \mathcal{A}_{1, \gamma}$ can be reconstructed from the $\delta \mathcal{R}_{1, \gamma}$ counterterm using only four-dimensional ingredients. However, it should be clear that, a priori, $\tilde{\mathbf{K}} \mathcal{A}_{1, \gamma}$ depends on the $(D-4)$-dimensional part of the loop numerator.
} 

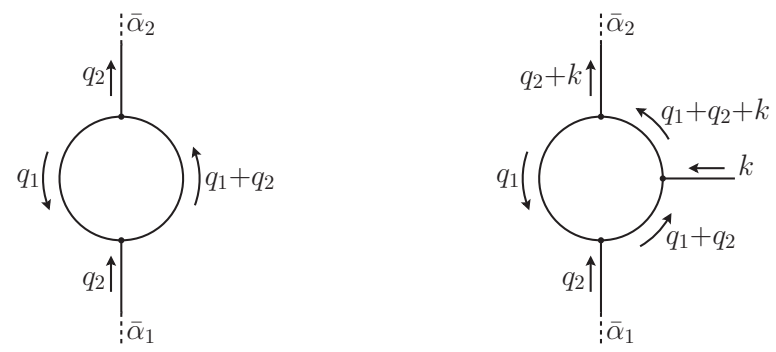

Figure 1. One-loop subtopologies that can give rise to non-logarithmic UV divergences.

\subsection{One-loop subdiagram in $D_{\mathrm{n}}=D$ dimensions}

Let us consider one-loop subdiagrams of the type shown in figure 1. The corresponding loop numerators have the form

$$
\overline{\mathcal{N}}^{\bar{\alpha}}\left(\bar{q}_{1}, \bar{q}_{2}\right)=\sum_{r=0}^{R} \overline{\mathcal{N}}_{\bar{\mu}_{1} \cdots \bar{\mu}_{r}}^{\bar{\alpha}}\left(\bar{q}_{2}\right) \bar{q}_{1}^{\bar{\mu}_{1}} \cdots \bar{q}_{1}^{\bar{\mu}_{r}}
$$

where $\bar{q}_{1}$ is the loop momentum of the subdiagram at hand, $\bar{q}_{2}$ is the external loop momentum, and the multi-index $\bar{\alpha}=\left(\bar{\alpha}_{1}, \bar{\alpha}_{2}\right)$ combines the two Lorentz/Dirac indices associated with the two $\bar{q}_{2}$-dependent external lines.

For what concerns UV poles and $\tilde{\mathcal{N}}$-contributions, as long as the dimensionality of $\bar{q}_{2}$ is the same in the loop numerator and denominator, the analysis of section 3.3 is applicable to the case at hand via naive extension of the external degrees of freedom from four to $D$ dimensions. More explicitly, the formulas (3.31)-(3.34) take the form,

$$
\overline{\mathbf{K}} \overline{\mathcal{A}}_{1, \gamma}^{\bar{\alpha}}\left(\bar{q}_{2}\right)=\mathbf{K} \overline{\mathcal{A}}_{1, \gamma}^{\bar{\alpha}}\left(\bar{q}_{2}\right)+\tilde{\mathbf{K}} \mathcal{A}_{1, \gamma}^{\bar{\alpha}}\left(\bar{q}_{2}\right)=\sum_{r=0}^{R} \overline{\mathcal{N}}_{\bar{\mu}_{1} \cdots \bar{\mu}_{r}}^{\overline{\bar{\alpha}}}\left(\bar{q}_{2}\right) \mathbf{K} T_{N}^{\bar{\mu}_{1} \cdots \bar{\mu}_{r}}\left(\bar{q}_{2}\right),
$$

with the UV divergent part

$$
\mathbf{K} \overline{\mathcal{A}}_{1, \gamma}^{\bar{\alpha}}\left(\bar{q}_{2}\right)=\sum_{r=0}^{R} \mathcal{N}_{\mu_{1} \cdots \mu_{r}}^{\bar{\alpha}}\left(\bar{q}_{2}\right) \mathbf{K} T_{N}^{\mu_{1} \cdots \mu_{r}}\left(\bar{q}_{2}\right)=-\delta Z_{1, \gamma}^{\bar{\alpha}}\left(\bar{q}_{2}\right),
$$

and the $\tilde{\mathcal{N}}$-part

$$
\tilde{\mathbf{K}} \mathcal{A}_{1, \gamma}^{\bar{\alpha}}\left(\bar{q}_{2}\right)=\sum_{r=0}^{R}\left[\overline{\mathcal{N}}_{\bar{\mu}_{1} \cdots \bar{\mu}_{r}}^{\bar{\alpha}}\left(\bar{q}_{2}\right)-\mathcal{N}_{\mu_{1} \cdots \mu_{r}}^{\bar{\alpha}}\left(\bar{q}_{2}\right)\right] \mathbf{K} T_{N}^{\bar{\mu}_{1} \cdots \bar{\mu}_{r}}\left(\bar{q}_{2}\right)=\delta \mathcal{R}_{1, \gamma}^{\bar{\alpha}}\left(\bar{q}_{2}\right) .
$$

The tensor integrals $T_{N}^{\bar{\mu}_{1} \cdots \bar{\mu}_{r}}\left(\bar{q}_{2}\right)$ are defined as in (3.10), and their $\bar{q}_{2}$-dependence originates entirely from the loop denominators. All quantities in (4.2)-(4.4), including the counterterms $\delta Z_{1, \gamma}^{\bar{\alpha}}\left(\bar{q}_{2}\right)$ and $\delta \mathcal{R}_{1, \gamma}^{\bar{\alpha}}\left(\bar{q}_{2}\right)$, are polynomials of degree $X$ in $\bar{q}_{2}$, and are related to the corresponding quantities in (3.31)-(3.34) through the replacements $q_{2} \rightarrow \bar{q}_{2}$ and $\alpha \rightarrow \bar{\alpha}$. For later convenience we also rewrite (3.34) as

$$
\tilde{\mathbf{K}} \mathcal{A}_{1, \gamma}^{\alpha}\left(q_{2}\right)=\sum_{r=0}^{R}\left[\overline{\mathcal{N}}_{\bar{\mu}_{1} \cdots \bar{\mu}_{r}}^{\alpha}\left(q_{2}\right)-\mathcal{N}_{\mu_{1} \cdots \mu_{r}}^{\alpha}\left(q_{2}\right)\right] \mathbf{K} T_{N}^{\bar{\mu}_{1} \cdots \bar{\mu}_{r}}\left(q_{2}\right)=\delta \mathcal{R}_{1, \gamma}^{\alpha}\left(q_{2}\right),
$$


where the dependence on $q_{2}$ and $\alpha$ is made explicit. Since (3.34) and (4.4) are free from UV poles, as long as $q_{2}$ is not integrated they differ only by terms of order $(D-4)$. More precisely,

$$
\tilde{\mathbf{K}} \overline{\mathcal{A}}_{1, \gamma}^{\bar{\alpha}}\left(\bar{q}_{2}\right)=\tilde{\mathbf{K}} \mathcal{A}_{1, \gamma}^{\alpha}\left(q_{2}\right)+\mathcal{O}\left(\varepsilon, \tilde{q}_{2}\right) .
$$

As an example of a one-loop diagram with $D$-dimensional external momentum, let us consider the massless photon selfenergy in QED,

$$
\overline{\mathcal{A}}_{1, \gamma}^{\bar{\alpha}_{1} \bar{\alpha}_{2}}\left(\bar{q}_{2}\right)=-\mathrm{i} e^{2} \int \mathrm{d} \bar{q}_{1} \frac{\operatorname{Tr}\left[\bar{\gamma}^{\bar{\alpha}_{1}} \not_{1} \bar{\gamma}^{\bar{\alpha}_{2}}\left(\bar{q}_{1}+\bar{q}_{2}\right)\right]}{\bar{q}_{1}^{2}\left(\bar{q}_{1}+\bar{q}_{2}\right)^{2}} .
$$

In this case, the quadratic UV divergence generates quadratic polynomials of the external momentum $\bar{q}_{2}$,

$$
\overline{\mathbf{K}} \overline{\mathcal{A}}_{1, \gamma}^{\bar{\alpha}_{1} \bar{\alpha}_{2}}\left(\bar{q}_{2}\right) \stackrel{\mathrm{MS}}{=} \frac{\mathrm{i} \alpha}{4 \pi}\left[-\frac{4}{3 \varepsilon}\left(\bar{q}_{2}^{2} g^{\bar{\alpha}_{1} \bar{\alpha}_{2}}-\bar{q}_{2}^{\bar{\alpha}_{1}} \bar{q}_{2}^{\bar{\alpha}_{2}}\right)+\frac{2}{3} \bar{q}_{2}^{2} g^{\bar{\alpha}_{1} \bar{\alpha}_{2}}\right],
$$

where the two terms between square brackets correspond, respectively, to the UV pole (4.3) and the rational $\tilde{\mathcal{N}}$-contribution (4.4). Note that for the examples discussed in this section we adopt the MS scheme, while the final results in section 6 are presented in the $\overline{\mathrm{MS}}$ scheme.

\subsection{One-loop subdiagram in $D_{\mathrm{n}}=4$ dimensions}

In order to identify the $\tilde{\mathcal{N}}$-contributions that originate from one-loop subdiagrams, in the following we compare the $D$-dimensional numerator (4.1) to its four-dimensional variant,

$$
\mathcal{N}^{\alpha}\left(q_{1}, q_{2}\right)=\sum_{r=0}^{R} \mathcal{N}_{\mu_{1} \cdots \mu_{r}}^{\alpha}\left(q_{2}\right) q_{1}^{\mu_{1}} \cdots q_{1}^{\mu_{r}}
$$

where all parts of the numerator, including the multi-index $\alpha$ and the external loop momentum $q_{2}$, are projected to four dimensions. At the amplitude level, in analogy with (4.2), the interplay of the numerator (4.9) with the UV poles that arise from the $\bar{q}_{1}$-integration results into

$$
\overline{\mathbf{K}} \mathcal{A}_{1, \gamma}^{\alpha}\left(q_{2}\right)=\mathbf{K} \mathcal{A}_{1, \gamma}^{\alpha}\left(q_{2}\right)=\sum_{r=0}^{R} \mathcal{N}_{\mu_{1} \cdots \mu_{r}}^{\alpha}\left(q_{2}\right) \mathbf{K} T_{N}^{\mu_{1} \cdots \mu_{r}}\left(\bar{q}_{2}\right),
$$

where the tensor integrals $T_{N}^{\mu_{1} \cdots \mu_{r}}\left(\bar{q}_{2}\right)$ depend on $\bar{q}_{2}$ since the external loop momentum is kept in $D$ dimensions in the loop denominator. The full pole contribution (4.10) can be split into two parts,

$$
\mathbf{K} \mathcal{A}_{1, \gamma}^{\alpha}\left(q_{2}\right)=-\delta Z_{1, \gamma}^{\alpha}\left(q_{2}\right)-\delta \tilde{Z}_{1, \gamma}^{\alpha}\left(\tilde{q}_{2}\right)
$$

where the first part reads

$$
-\delta Z_{1, \gamma}^{\alpha}\left(q_{2}\right)=\sum_{r=0}^{R} \mathcal{N}_{\mu_{1} \cdots \mu_{r}}^{\alpha}\left(q_{2}\right) \mathbf{K} T_{N}^{\mu_{1} \cdots \mu_{r}}\left(q_{2}\right)
$$

and corresponds to the standard UV counterterm (4.3) with $\left(\bar{\alpha}, \bar{q}_{2}\right)$ replaced by $\left(\alpha, q_{2}\right)$ throughout. The remnant part originates from the $(D-4)$-dimensional part of $\bar{q}_{2}$ in the 
denominator of the one-loop subdiagram and reads

$$
-\delta \tilde{Z}_{1, \gamma}^{\alpha}\left(\tilde{q}_{2}\right)=\sum_{r=0}^{R} \mathcal{N}_{\mu_{1} \cdots \mu_{r}}^{\alpha}\left(q_{2}\right) \Delta K_{N}^{\mu_{1} \cdots \mu_{r}}\left(\tilde{q}_{2}\right),
$$

with

$$
\Delta K_{N}^{\mu_{1} \cdots \mu_{r}}\left(\tilde{q}_{2}\right)=\mathbf{K} T_{N}^{\mu_{1} \cdots \mu_{r}}\left(\bar{q}_{2}\right)-\mathbf{K} T_{N}^{\mu_{1} \cdots \mu_{r}}\left(q_{2}\right) .
$$

In renormalisable theories, where the maximum degree of divergence of one-loop integrals is $X=2$, the tensor-integral poles in (4.14) are at most quadratic in $\bar{q}_{2}$. Their general form is

$$
\mathbf{K} T_{N}^{\mu_{1} \cdots \mu_{r}}\left(\bar{q}_{2}\right) \stackrel{\mathrm{MS}}{=} \frac{1}{\varepsilon}\left[A^{\mu_{1} \cdots \mu_{r}}+B_{\bar{\nu}_{1}}^{\mu_{1} \cdots \mu_{r}} \bar{q}_{2}^{\bar{\nu}_{1}}+C_{\bar{\nu}_{1} \bar{\nu}_{2}}^{\mu_{1} \cdots \mu_{r}} \bar{q}_{2}^{\bar{\nu}_{1}} \bar{q}_{2}^{\bar{\nu}_{2}}\right]
$$

where the tensors $A, B$ and $C$ consist of combinations of the other four-dimensional external momenta $p_{k}$ and metric tensors, which carry four-dimensional indices $\mu_{i}$ or $D$-dimensional indices $\bar{\nu}_{j}$. In (4.14) the $\bar{q}_{2}$-independent contribution associated with the tensor $A$ cancels, and for integrals with degree of divergence $X \leq 1$, where the tensor $C$ associated with the quadratic $\bar{q}_{2}$ terms vanishes, we have

$$
\left.\Delta K_{N}^{\mu_{1} \cdots \mu_{r}}\left(\tilde{q}_{2}\right)\right|_{X \leq 1} \stackrel{\text { MS }}{=} \frac{1}{\varepsilon} B_{\bar{\nu}_{1}}^{\mu_{1} \cdots \mu_{r}} \tilde{q}_{2}^{\bar{\nu}_{1}}=0 .
$$

This cancellation is due to the fact that the tensor $B$ carries a single $D$-dimensional index, which can lead only to terms of the form $p_{k} \cdot \tilde{q}_{2}=0$ or $g_{\tilde{\nu}_{1}}^{\mu_{i}} \tilde{q}_{2}^{\bar{\nu}_{1}}=\tilde{q}_{2}^{\mu_{i}}=0$. Thus (4.14) is non-vanishing only for quadratically divergent integrals. In this case

$$
\begin{aligned}
\left.\Delta K_{N}^{\mu_{1} \cdots \mu_{r}}\left(\tilde{q}_{2}\right)\right|_{X=2} & \stackrel{\mathrm{MS}}{=} \frac{1}{\varepsilon} C_{\bar{\nu}_{1} \bar{\nu}_{2}}^{\mu_{1} \cdots \mu_{r}}\left(\bar{q}_{2}^{\bar{\nu}_{1}} \bar{q}_{2}^{\bar{\nu}_{2}}-q_{2}^{\nu_{1}} q_{2}^{\nu_{2}}\right) \\
& =\frac{1}{\varepsilon} C_{00}^{\mu_{1} \cdots \mu_{r}} g_{\bar{\nu}_{1} \bar{\nu}_{2}}\left(\bar{q}_{2}^{\bar{\nu}_{1}} \bar{q}_{2}^{\bar{\nu}_{2}}-q_{2}^{\nu_{1}} q_{2}^{\nu_{2}}\right)=C_{00}^{\mu_{1} \cdots \mu_{r}} \frac{\tilde{q}_{2}^{2}}{\varepsilon},
\end{aligned}
$$

where we have split the tensor $C$ into a part $C_{00}^{\mu_{1} \ldots \mu_{r}} g_{\bar{\nu}_{1} \bar{\nu}_{2}}$ and a remaining part that does not contribute to (4.17) since one or both $\bar{\nu}_{i}$ indices are either carried by a four-dimensional external momentum or by a $g_{\bar{\nu}_{i}}^{\mu_{j}}$ tensor. Based on (4.16)-(4.17) we conclude that in renormalisable theories the extra counterterms (4.13) are required only for quadratically divergent selfenergies, and their general form is

$$
\delta \tilde{Z}_{1, \gamma}^{\alpha}\left(\tilde{q}_{2}\right) \stackrel{\mathrm{MS}}{=} v^{\alpha} \frac{\tilde{q}_{2}^{2}}{\varepsilon},
$$

where $v^{\alpha}$ is independent of $q_{2}$. Such extra counterterms should be regarded as an extension of the usual UV counterterms for the case of one-loop integrals with numerators in $D_{\mathrm{n}}=4$ and denominators in $D=4-2 \varepsilon$ dimensions. Note that upon integration over $\bar{q}_{2}$ the terms of order $\tilde{q}_{2}^{2} / \varepsilon$ in (4.18) result into two-loop contributions of order $\varepsilon^{0}$.

As an example of the UV poles of a one-loop diagram in $D_{\mathrm{n}}=4$ dimensions, let us consider again the massless photon selfenergy in QED,

$$
\mathcal{A}_{1, \gamma}^{\alpha_{1} \alpha_{2}}\left(q_{2}\right)=-\mathrm{i} e^{2} \int \mathrm{d} \bar{q}_{1} \frac{\operatorname{Tr}\left[\gamma^{\alpha_{1}} \not_{1} \gamma^{\alpha_{2}}\left(\not_{1}+q_{2}\right)\right]}{\bar{q}_{1}^{2}\left(\bar{q}_{1}+\bar{q}_{2}\right)^{2}},
$$


where

$$
\mathbf{K} \mathcal{A}_{1, \gamma}^{\alpha_{1} \alpha_{2}}\left(q_{2}\right) \stackrel{\mathrm{MS}}{=} \frac{\mathrm{i} \alpha}{4 \pi}\left[-\frac{4}{3 \varepsilon}\left(q_{2}^{2} g^{\alpha_{1} \alpha_{2}}-q_{2}^{\alpha_{1}} q_{2}^{\alpha_{2}}\right)-\frac{2}{3} \frac{\tilde{q}_{2}^{2}}{\varepsilon} g^{\alpha_{1} \alpha_{2}}\right] .
$$

Here the two terms between square brackets correspond, respectively, to the standard UV counterterm (4.12) and the $\mathcal{O}\left(\tilde{q}_{2} / \varepsilon\right)$ remnant (4.13).

\subsection{Relating renormalised one-loop subdiagrams in $D_{\mathrm{n}}=D$ and $D_{\mathrm{n}}=4$}

In this section we extend the identity (1.3) to one-loop amplitudes with $D$-dimensional external momenta. As a starting point we consider the amplitude of a renormalised subdiagram in $D_{\mathrm{n}}=D$ dimensions,

$$
\mathbf{R} \overline{\mathcal{A}}_{1, \gamma}^{\bar{\alpha}}\left(\bar{q}_{2}\right)=(1-\mathbf{K}) \overline{\mathcal{A}}_{1, \gamma}^{\bar{\alpha}}\left(\bar{q}_{2}\right)=\overline{\mathcal{A}}_{1, \gamma}^{\bar{\alpha}}\left(\bar{q}_{2}\right)+\delta Z_{1, \gamma}^{\bar{\alpha}}\left(\bar{q}_{2}\right),
$$

and we relate it to corresponding quantities in $D_{\mathrm{n}}=4$ dimensions by means of rational terms. To this end, using (4.2) as an auxiliary subtraction term in $D_{\mathrm{n}}=D$ we define the subtracted amplitude

$$
\overline{\mathcal{A}}_{1, \gamma}^{\bar{\alpha}}\left(\bar{q}_{2}\right)-\overline{\mathbf{K}} \overline{\mathcal{A}}_{1, \gamma}^{\bar{\alpha}}\left(\bar{q}_{2}\right)=\sum_{r=0}^{R} \overline{\mathcal{N}}_{\bar{\mu}_{1} \cdots \bar{\mu}_{r}}^{\bar{\alpha}}\left(\bar{q}_{2}\right)\left[T_{N}^{\bar{\mu}_{1} \cdots \bar{\mu}_{r}}\left(\bar{q}_{2}\right)-\mathbf{K} T_{N}^{\bar{\mu}_{1} \cdots \bar{\mu}_{r}}\left(\bar{q}_{2}\right)\right] .
$$

Similarly, using (4.10) as a subtraction term in $D_{\mathrm{n}}=4$ we define

$$
\mathcal{A}_{1, \gamma}^{\alpha}\left(q_{2}\right)-\mathbf{K} \mathcal{A}_{1, \gamma}^{\alpha}\left(q_{2}\right)=\sum_{r=0}^{R} \mathcal{N}_{\mu_{1} \cdots \mu_{r}}^{\alpha}\left(q_{2}\right)\left[T_{N}^{\mu_{1} \cdots \mu_{r}}\left(\bar{q}_{2}\right)-\mathbf{K} T_{N}^{\mu_{1} \cdots \mu_{r}}\left(\bar{q}_{2}\right)\right] .
$$

By construction, in both cases the subtraction terms cancel the full pole contribution at the level of tensor integrals. Thus the terms between square brackets in (4.22) and (4.23) are free from $1 / \varepsilon$ poles and differ only by terms of $\mathcal{O}(\varepsilon)$. As a consequence, also the whole subtracted amplitudes differ only by terms of order $(\varepsilon)$. More explicitly,

$$
\overline{\mathcal{A}}_{1, \gamma}^{\bar{\alpha}}\left(\bar{q}_{2}\right)-\overline{\mathbf{K}} \overline{\mathcal{A}}_{1, \gamma}^{\bar{\alpha}}\left(\bar{q}_{2}\right)=\mathcal{A}_{1, \gamma}^{\alpha}\left(q_{2}\right)-\mathbf{K} \mathcal{A}_{1, \gamma}^{\alpha}\left(q_{2}\right)+\mathcal{O}\left(\varepsilon, \tilde{q}_{2}\right) .
$$

This identity can be turned into a relation between renormalised amplitudes by splitting $\overline{\mathbf{K}}$ into $\mathbf{K}+\tilde{\mathbf{K}}$ as in (4.2), using (4.6) for the $\tilde{\mathbf{K}}$ part, and shifting the latter to the l.h.s. In this way one arrives at

$$
(1-\mathbf{K}) \overline{\mathcal{A}}_{1, \gamma}^{\bar{\alpha}}\left(\bar{q}_{2}\right)=(1-\mathbf{K}+\tilde{\mathbf{K}}) \mathcal{A}_{1, \gamma}^{\alpha}\left(q_{2}\right)+\mathcal{O}\left(\varepsilon, \tilde{q}_{2}\right) .
$$

Finally, expressing the $\mathbf{K}$ and $\tilde{\mathbf{K}}$ terms through the corresponding UV and rational counterterms introduced in (4.3), (4.5) and (4.11) leads to

$$
\mathbf{R} \overline{\mathcal{A}}_{1, \gamma}^{\bar{\alpha}}\left(\bar{q}_{2}\right)=\mathcal{A}_{1, \gamma}^{\alpha}\left(q_{2}\right)+\delta Z_{1, \gamma}^{\alpha}\left(q_{2}\right)+\delta \tilde{Z}_{1, \gamma}^{\alpha}\left(\tilde{q}_{2}\right)+\delta \mathcal{R}_{1, \gamma}^{\alpha}\left(q_{2}\right)+\mathcal{O}\left(\varepsilon, \tilde{q}_{2}\right) .
$$

This identity relates the UV-renormalised amplitude in $D$ dimensions, on the l.h.s., to the corresponding amplitude with four-dimensional numerator plus three counterterms: the usual UV counterterm $\delta Z_{1, \gamma}^{\alpha}$ with $\alpha$ and $q_{2}$ in four dimensions, its $\mathcal{O}\left(\tilde{q}_{2}^{2} / \varepsilon\right)$ extension 


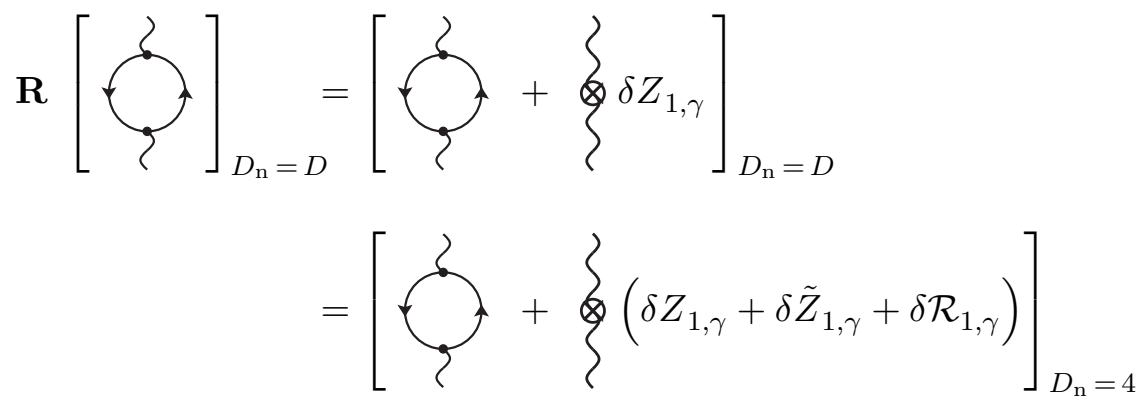

Figure 2. Graphical representation of (4.21) for a renormalised QED selfenergy in $D_{\mathrm{n}}=D$ numerator dimensions and the relation (4.26) to its counterpart in $D_{\mathrm{n}}=4$ dimensions. The indices $\alpha=\left(\alpha_{1}, \alpha_{2}\right)$, the external loop momentum $q_{2}$ and terms of $\mathcal{O}(\varepsilon)$ are implicitly understood.

$\delta \tilde{Z}_{1, \gamma}^{\alpha}$, defined in (4.13)-(4.18), and the rational term $\delta \mathcal{R}_{1, \gamma}^{\alpha}$, which compensates for the missing $\tilde{\mathcal{N}}$-part of the loop numerator. At two loops, the identity (4.26) will play a key role for the extraction of UV poles and $\tilde{\mathcal{N}}$-contributions that arise from divergent one-loop subdiagrams (see section 5).

The renormalised amplitude (4.21) in $D_{\mathrm{n}}=D$ and the identity (4.26) are illustrated in figure 2 for the case of a QED selfenergy.

\section{Rational terms at two loops}

In this section we derive a general formula for the reconstruction of the $\tilde{\mathcal{N}}$-contributions of two-loop amplitudes in any renormalisable theory. We also present an explicit recipe for the calculation of the relevant process-independent two-loop counterterms in terms of tadpole integrals.

\subsection{Notation for two-loop diagrams and subdiagrams}

Irreducible two-loop diagrams involve propagators that depend on the loop momenta $q_{1}$, $q_{2}$ and $q_{3}=-q_{1}-q_{2}$. Their generic structure is illustrated in figure 3 and consists of three chains, $\mathcal{C}_{1}, \mathcal{C}_{2}, \mathcal{C}_{3}$, that are connected to each other by two vertices, $\mathcal{V}_{0}, \mathcal{V}_{1}$. Each chain $\mathcal{C}_{i}$ includes a certain number $N_{i}$ of propagators that depend on the loop momentum $q_{i}$ and the $N_{i}-1$ vertices that connect them to each other and to external lines. The two-loop integral associated with a generic two-loop diagram $\Gamma$ has the form ${ }^{10}$

$$
\overline{\mathcal{A}}_{2, \Gamma}=\int \mathrm{d} \bar{q}_{1} \int \mathrm{d} \bar{q}_{2} \int \mathrm{d} \bar{q}_{3} \delta\left(\bar{q}_{1}+\bar{q}_{2}+\bar{q}_{3}\right) \frac{\bar{\Gamma}^{\bar{\alpha}_{1} \bar{\alpha}_{2} \bar{\alpha}_{3}}\left(\bar{q}_{1}, \bar{q}_{2}, \bar{q}_{3}\right) \overline{\mathcal{N}}_{\bar{\alpha}_{1}}^{(1)}\left(\bar{q}_{1}\right) \overline{\mathcal{N}}_{\bar{\alpha}_{2}}^{(2)}\left(\bar{q}_{2}\right) \overline{\mathcal{N}}_{\bar{\alpha}_{3}}^{(3)}\left(\bar{q}_{3}\right)}{\mathcal{D}^{(1)}\left(\bar{q}_{1}\right) \mathcal{D}^{(2)}\left(\bar{q}_{2}\right) \mathcal{D}^{(3)}\left(\bar{q}_{3}\right)}
$$

where each chain $\mathcal{C}_{i}$ contributes through the corresponding set of loop denominators,

$$
\mathcal{D}^{(i)}\left(\bar{q}_{i}\right)=D_{0}^{(i)}\left(\bar{q}_{i}\right) \cdots D_{N_{i}-1}^{(i)}\left(\bar{q}_{i}\right), \quad \text { with } \quad D_{a}^{(i)}\left(\bar{q}_{i}\right)=\left(\bar{q}_{i}+p_{i a}\right)^{2}-m_{i a}^{2},
$$

\footnotetext{
${ }^{10}$ This two-particle irreducible amplitude corresponds to $\overline{\mathcal{A}}_{2, \Gamma}^{\sigma_{1} \ldots \sigma_{N}}$ in (2.15), but here and in the following the external indices $\sigma_{1} \ldots \sigma_{N}$ are kept implicit.
} 


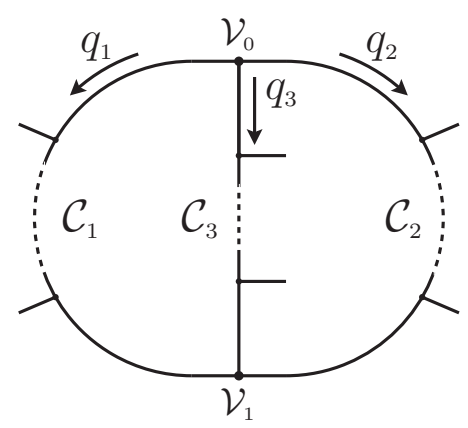

Figure 3. A generic irreducible two-loop diagram consists of two vertices, $\mathcal{V}_{0}, \mathcal{V}_{1}$, that connect three chains, $\mathcal{C}_{1}, \mathcal{C}_{2}, \mathcal{C}_{3}$, which contain, respectively, all propagators that depend on the loop momenta $q_{1}, q_{2}, q_{3}=-q_{1}-q_{2}$, as well as all vertices that connect the propagators depending on the same loop momentum. Note that in general the loop momenta that flow out of the $\mathcal{V}_{0}$ vertex should be $q_{i}+p_{i 0}$, but for triple vertices one can set $p_{i 0}=0$ as in the above graph. In contrast, if $\mathcal{V}_{0}$ is a quartic vertex, momentum conservation requires non-vanishing $p_{i 0}$ momenta with $\sum_{i} p_{i 0}$ equal to the external momentum that flows into $\mathcal{V}_{0}$.

and a loop numerator part $\overline{\mathcal{N}}_{\bar{\alpha}_{i}}^{(i)}\left(\bar{q}_{i}\right)$. The latter carries a multi-index $\bar{\alpha}_{i} \equiv\left(\bar{\alpha}_{i 1}, \bar{\alpha}_{i 2}\right)$ that

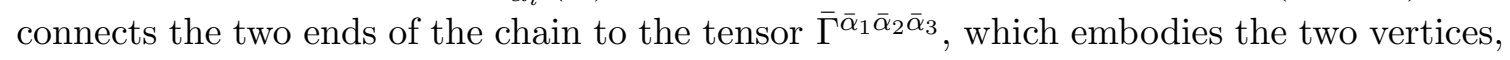
$\mathcal{V}_{0}$ and $\mathcal{V}_{1}$. Integrating $(5.1)$ over $\bar{q}_{3}$ yields

$$
\overline{\mathcal{A}}_{2, \Gamma}=\int \mathrm{d} \bar{q}_{1} \int \mathrm{d} \bar{q}_{2} \frac{\overline{\mathcal{N}}\left(\bar{q}_{1}, \bar{q}_{2}\right)}{\mathcal{D}^{(1)}\left(\bar{q}_{1}\right) \mathcal{D}^{(2)}\left(\bar{q}_{2}\right) \mathcal{D}^{(3)}\left(-\bar{q}_{1}-\bar{q}_{2}\right)}
$$

where $\overline{\mathcal{N}}\left(\bar{q}_{1}, \bar{q}_{2}\right)$ corresponds to the numerator of $(5.1)$ at $\bar{q}_{3}=-\bar{q}_{1}-\bar{q}_{2}$. Similarly as in (3.4)-(3.6) the two-loop numerator can be split into four- and $(D-4)$-dimensional parts as

$$
\overline{\mathcal{N}}\left(\bar{q}_{1}, \bar{q}_{2}\right)=\mathcal{N}\left(q_{1}, q_{2}\right)+\tilde{\mathcal{N}}\left(\bar{q}_{1}, \bar{q}_{2}\right)
$$

where

$$
\mathcal{N}\left(q_{1}, q_{2}\right)=\left.\overline{\mathcal{N}}\left(\bar{q}_{1}, \bar{q}_{2}\right)\right|_{\bar{g} \rightarrow g, \bar{\gamma} \rightarrow \gamma, \bar{q}_{1} \rightarrow q_{1}, \bar{q}_{2} \rightarrow q_{2}}
$$

and

$$
\tilde{\mathcal{N}}\left(\bar{q}_{1}, \bar{q}_{2}\right)=\mathcal{O}\left(\varepsilon, \tilde{q}_{1}, \tilde{q}_{2}\right)
$$

The main goal of this paper is to derive a general formula for the reconstruction of all relevant $\tilde{\mathcal{N}}$-contributions of UV origin, i.e. all terms of order $\varepsilon^{-1}$ and $\varepsilon^{0}$ that originate form the interplay the $(D-4)$-dimensional part of the numerator (5.6) with single and double $1 / \varepsilon$ poles of UV type.

The analysis of $\tilde{\mathcal{N}}$-contributions beyond one loop requires a careful treatment of subdiagrams and their UV divergences. At two loops, each diagram $\Gamma$ involves three subdiagrams, $\gamma_{1}, \gamma_{2}, \gamma_{3}$, where $\gamma_{i}$ results from $\Gamma$ by cutting the chain $\mathcal{C}_{i}$. More precisely, each partition $i \mid j k$ of 123 corresponds to a subdiagram $\gamma_{i}$ that involves the chains $\mathcal{C}_{j}, \mathcal{C}_{k}$ and the vertices 
$\mathcal{V}_{0}, \mathcal{V}_{1}$. Its amplitude reads

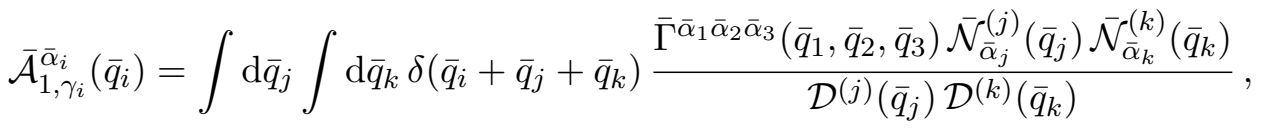

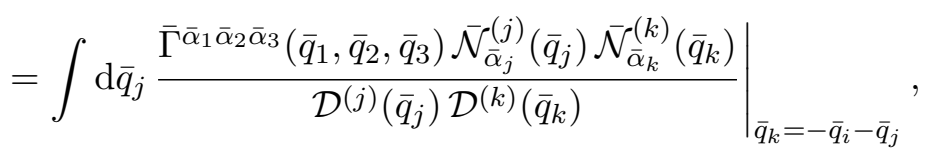

where $\bar{q}_{i}$ plays the role of external momentum for the subdiagram $\gamma_{i}$.

For each subdiagram $\gamma_{i}$ of $\Gamma$ we define its complement $\Gamma / \gamma_{i}$ as the one-loop diagram that involves only the chain $\mathcal{C}_{i}$ and results form $\Gamma$ by shrinking the chains $\mathcal{C}_{j}, \mathcal{C}_{k}$ to a vertex. Thus the full two-loop diagram $\Gamma$ can be expressed as the insertion of the subdiagram $\gamma_{i}$ into its complement $\Gamma / \gamma_{i}$. For such insertions we use the notation

$$
\overline{\mathcal{A}}_{2, \Gamma}=\overline{\mathcal{A}}_{1, \gamma_{i}} \cdot \overline{\mathcal{A}}_{1, \Gamma / \gamma_{i}}=\int \mathrm{d} \bar{q}_{i} \overline{\mathcal{A}}_{1, \gamma_{i}}^{\bar{\alpha}_{i}}\left(\bar{q}_{i}\right) \frac{\overline{\mathcal{N}}_{\bar{\alpha}_{i}}^{(i)}\left(\bar{q}_{i}\right)}{\mathcal{D}^{(i)}\left(\bar{q}_{i}\right)}
$$

where the dot product involves the integration over the loop momentum $q_{i}$ and the summation over the multi-index $\alpha_{i}$, as defined on the r.h.s.

\subsection{Power counting and structure of UV divergences}

Divergences of UV type can be easily identified through naive power counting, i.e. by counting the maximum power in the loop momenta $q_{i}$ at the integrand level. For the analysis of two-loop divergences we count the powers of loop momenta originating from the loop chains $\mathcal{C}_{1}, \mathcal{C}_{2}, \mathcal{C}_{3}$ and the connecting vertices $\mathcal{V}_{0}, \mathcal{V}_{1}$ as follows. For a two-loop diagram $\Gamma$ we define $U_{i}(\Gamma)$ as the maximum power of the full chain $\mathcal{C}_{i}$ in the corresponding loop momentum $q_{i}$ at $q_{i} \rightarrow \infty$. In QCD we have

$$
U_{i}(\Gamma)=-n_{\text {prop }}^{q q, i}-2 n_{\text {prop }}^{g g, i}-2 n_{\text {prop }}^{u u, i}+n_{\text {vert }}^{g g g, i}+n_{\text {vert }}^{u u g, i},
$$

where the various terms on the r.h.s. represent the numbers of propagators $\left(n_{\text {prop }}\right)$ and vertices $\left(n_{\text {vert }}\right)$ involving quarks $(q)$, gluons $(g)$ and ghosts $(u)$ along the chain $\mathcal{C}_{i}$. The loop-momentum power of the vertices $V_{a}$ that connect the three loop chains is denoted $Y_{a}(\Gamma)$. In QED we have $Y_{a}(\Gamma)=0$, while in QCD

$$
Y_{a}(\Gamma)= \begin{cases}1 & \text { if } V_{a} \text { is a trilinear gluon vertex } \\ 0 & \text { otherwise }\end{cases}
$$

The loop momenta associated with ghost-gluon vertices can always be assigned to a unique chain $\mathcal{C}_{i}$, also in case of an intersection vertex $\mathcal{V}_{a}$. Thus $g u \bar{u}$ vertices should be accounted for through the corresponding counter $n_{\mathrm{vert}}^{\text {uug,i}}$ in $U_{i}(\Gamma)$ and not through $Y_{a}(\Gamma)$.

The simplest divergences of two-loop diagrams $\Gamma$ are the ones arising from their oneloop subdiagrams $\gamma_{i}$. They can be identified by means of the degree of subdivergence

$$
X\left(\gamma_{i}\right)=X_{j k}(\Gamma)=4+U_{j}(\Gamma)+U_{k}(\Gamma)+\sum_{a=0}^{1} Y_{a}(\Gamma),
$$


where $i \mid j k$ is a partition of 123 . When $X\left(\gamma_{i}\right) \geq 0$ the subdiagram $\gamma_{i}$ leads to a UV pole that arises from the region where $q_{j}, q_{k} \rightarrow \infty$ with $q_{i}$ fixed. Upon subtraction of all subdivergences, two-loop diagrams can involve residual local divergences. This kind of divergences originate from the region where $q_{1}, q_{2}, q_{3} \rightarrow \infty$ simultaneously. They can be identified by means of the global degree of divergence

$$
X(\Gamma)=8+\sum_{i=1}^{3} U_{i}(\Gamma)+\sum_{a=0}^{1} Y_{a}(\Gamma)
$$

which corresponds to the total loop-momentum power of the full two-loop diagram. Diagrams with $X(\Gamma) \geq 0$ will be referred to as globally divergent diagrams. Such diagrams can involve both subdivergences and residual local divergences. Instead, diagrams with $X(\Gamma)<0$ are free from local divergences and in renormalisable theories they involve at most one subdivergence, i.e.

$$
X(\Gamma)<0 \Rightarrow X\left(\gamma_{l}\right)<0 \quad \text { for at least two subdiagrams } \gamma_{l} .
$$

This well-known property ${ }^{11}$ will be exploited in section 5.5 in order to demonstrate that $\tilde{\mathcal{N}}$-contributions at two loops can be reconstructed by means of universal local counterterms.

\subsection{Structure of UV poles at two loops}

In general, two-loop amplitudes involve one-loop subdivergences and additional local divergences. The renormalisation of these two kinds of divergences can be schematically written in the form

$$
\mathbf{R} \overline{\mathcal{A}}_{2, \Gamma}=\overline{\mathcal{A}}_{2, \Gamma}-\mathbf{K}_{\mathrm{sub}} \overline{\mathcal{A}}_{2, \Gamma}-\mathbf{K}_{\mathrm{loc}} \overline{\mathcal{A}}_{2, \Gamma} .
$$

Here the $\mathbf{K}_{\text {sub }}$ operator extracts the divergences that result from the $\overline{\mathrm{MS}}$ poles of the three subdiagrams,

$$
\mathbf{K}_{\mathrm{sub}} \overline{\mathcal{A}}_{2, \Gamma}=\sum_{i=1}^{3}\left(\mathbf{K} \overline{\mathcal{A}}_{1, \gamma_{i}}\right) \cdot \overline{\mathcal{A}}_{1, \Gamma / \gamma_{i}}=-\sum_{i=1}^{3} \int \mathrm{d} \bar{q}_{i} \delta Z_{1, \gamma_{i}}^{\bar{\alpha}_{i}}\left(\bar{q}_{i}\right) \frac{\overline{\mathcal{N}}_{\bar{\alpha}_{i}}^{(i)}\left(\bar{q}_{i}\right)}{\mathcal{D}^{(i)}\left(\bar{q}_{i}\right)} .
$$

\footnotetext{
${ }^{11}$ The fact that $X(\Gamma)<0$ implies at most one subdivergence can be demonstrated by combining $(5.11)$ and (5.12) such as to obtain the following relation between the global degree of divergence $X(\Gamma)$ and the sum of the degrees of divergence of two arbitrary subdiagrams,

$$
X\left(\gamma_{j}\right)+X\left(\gamma_{k}\right)=X(\Gamma)+\tilde{X}_{i}(\Gamma)
$$

with

$$
\tilde{X}_{i}(\Gamma)=U_{i}(\Gamma)+Y_{0}(\Gamma)+Y_{1}(\Gamma) .
$$

The latter quantity describes the total $q_{i}$-power of the $N_{i}$ propagators of the chain $\mathcal{C}_{i}$ together with all (internal and external) $N_{i}+1$ vertices to which they are connected. In renormalisable theories, each of the $N_{i}$ combinations of a propagator with a neighbouring vertex contributes with power -1 or less, while the remaining extra vertex contributes with power +1 or less. Therefore

$$
\tilde{X}_{i}(\Gamma) \leq-N_{i}+1 \leq 0 \quad \text { for } \quad N_{i} \geq 1 .
$$

Combining (5.16) with (5.14) yields the following lower bound for the global degree of divergence,

$$
X(\Gamma) \geq X\left(\gamma_{j}\right)+X\left(\gamma_{k}\right),
$$

which implies, consistently with (5.13), that two or more subdivergences can occur only if $X(\Gamma)>0$.
} 


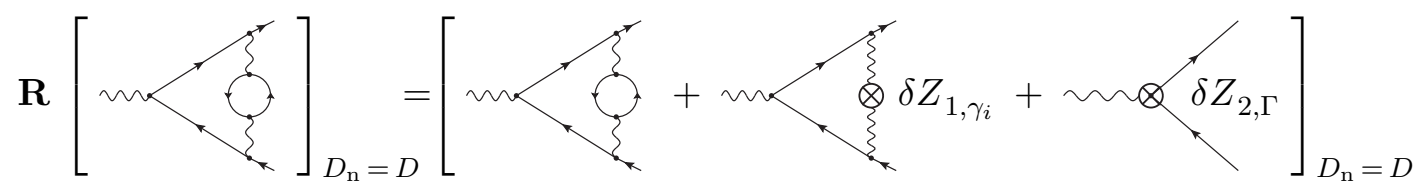

Figure 4. Graphical representation of the renormalisation formula (5.18) for a two-loop QED diagram with a single subdivergence.

The one-loop $\overline{\mathrm{MS}}$ counterterms $\delta Z_{1, \gamma_{i}}\left(\bar{q}_{i}\right)$ are local, i.e. they are polynomials in $\bar{q}_{i}$, but their insertion into two-loop diagrams gives rise to non-local terms. After subtraction of all oneloop subdivergences, two-loop diagrams with $X(\Gamma) \geq 0$ still involve local divergences. The corresponding $\overline{\mathrm{MS}}$ poles are extracted through the operator $\mathbf{K}_{\text {loc }}$, which is defined as

$$
\mathbf{K}_{\mathrm{loc}} \overline{\mathcal{A}}_{2, \Gamma}=\mathbf{K}\left(1-\mathbf{K}_{\mathrm{sub}}\right) \overline{\mathcal{A}}_{2, \Gamma}=-\delta Z_{2, \Gamma}
$$

where $\delta Z_{2, \Gamma}$ represents the two-loop UV counterterm for the diagram at hand.

Note that the renormalisation operator $\mathbf{R}$ and the associated operators $\mathbf{K}_{\text {sub }}$ and $\mathbf{K}_{\text {loc }}$ should be understood as linear operators. In particular, when a two-loop diagram $\Gamma$ is split into a sum of contributions $\Gamma_{\sigma}$, the $\mathbf{K}_{\text {sub }}$ operator fulfils

$$
\mathbf{K}_{\text {sub }}\left(\sum_{\sigma} \overline{\mathcal{A}}_{2, \Gamma_{\sigma}}\right)=\sum_{\sigma} \mathbf{K}_{\text {sub }} \overline{\mathcal{A}}_{2, \Gamma_{\sigma}},
$$

with

$$
\mathbf{K}_{\mathrm{sub}} \overline{\mathcal{A}}_{2, \Gamma_{\sigma}}=\sum_{i=1}^{3}\left(\mathbf{K} \overline{\mathcal{A}}_{1, \gamma_{\sigma i}}\right) \cdot \overline{\mathcal{A}}_{1, \Gamma_{\sigma} / \gamma_{\sigma i}} .
$$

Here $\gamma_{\sigma i}$ denotes the $i^{\text {th }}$ subdiagram associated with the contribution $\Gamma_{\sigma}$, and $\mathbf{K} \overline{\mathcal{A}}_{1, \gamma_{\sigma i}}$ is the corresponding UV pole.

The structure of the renormalisation formula (5.18) is illustrated in figure 4 for the case of a two-loop QED diagram with a single subdivergence.

\subsection{Structure of rational parts at two loops}

The main goal of this paper is to derive a general formula that relates renormalised twoloop amplitudes in $D_{\mathrm{n}}=D$ dimensions to corresponding amplitudes in $D_{\mathrm{n}}=4$ dimensions by means of rational counterterms. This formula will be derived in section 5.5, and in the following we anticipate its general structure, which reads

$$
\mathbf{R} \overline{\mathcal{A}}_{2, \Gamma}=\mathcal{A}_{2, \Gamma}+\left(\tilde{\mathbf{K}}_{\mathrm{sub}}-\mathbf{K}_{\mathrm{sub}}\right) \mathcal{A}_{2, \Gamma}+\left(\tilde{\mathbf{K}}_{\mathrm{loc}}-\mathbf{K}_{\mathrm{loc}}\right) \mathcal{A}_{2, \Gamma} .
$$

Here the subtraction of subdivergences and local two-loop divergences is implemented through the operators $\mathbf{K}_{\text {sub }}$ and $\mathbf{K}_{\text {loc }}$ in a similar way as in (5.18), but such operators are supplemented by the $\tilde{\mathbf{K}}_{\text {sub }}$ and $\tilde{\mathbf{K}}_{\text {loc }}$ operators, which reconstruct the $\tilde{\mathcal{N}}$-contributions that originate form the respective types of divergences. Similarly as for $\mathbf{K}_{\text {sub }}$ and $\mathbf{K}_{\text {loc }}$, also $\tilde{\mathbf{K}}_{\text {sub }}$ and $\tilde{\mathbf{K}}_{\text {loc }}$ should be understood as linear operators in the sense of (5.21)-(5.22). 
$\mathbf{R}\left[\sum_{D_{\mathrm{n}}=D}=\right.$

$=\left[m\left\{\sum_{\xi}+m\left(\delta Z_{1, \gamma_{i}}+\delta \tilde{Z}_{1, \gamma_{i}}+\delta \mathcal{R}_{1, \gamma_{i}}\right)+m \delta\left(\delta Z_{2, \Gamma}+\delta \mathcal{R}_{2, \Gamma}\right)\right]_{D_{\mathrm{n}}=4}\right.$

Figure 5. Graphical representation of the master formula (5.23), see also (1.5), for a globally divergent two-loop QED diagram with a single subdivergence.

According to our analysis in section 4 , the $\mathbf{K}_{\text {sub }}$ operator needs to be defined as

$$
\mathbf{K}_{\mathrm{sub}} \mathcal{A}_{2, \Gamma}=\sum_{i=1}^{3}\left(\mathbf{K} \mathcal{A}_{1, \gamma_{i}}\right) \cdot \mathcal{A}_{1, \Gamma / \gamma_{i}}=-\sum_{i=1}^{3} \int \mathrm{d} \bar{q}_{i}\left[\delta Z_{1, \gamma_{i}}^{\alpha_{i}}\left(q_{i}\right)+\delta \tilde{Z}_{1, \gamma_{i}}^{\alpha_{i}}\left(\tilde{q}_{i}\right)\right] \frac{\mathcal{N}_{\alpha_{i}}^{(i)}\left(q_{i}\right)}{\mathcal{D}^{(i)}\left(\bar{q}_{i}\right)}
$$

where the extended counterterms $\delta Z_{1, \gamma_{i}}+\delta \tilde{Z}_{1, \gamma_{i}}$ guarantee the consistent subtraction of UV poles in $D_{\mathrm{n}}=4$ dimensions (4.11). The $\tilde{\mathcal{N}}$-contributions stemming from subdivergences, see (4.5), are reconstructed by

$$
\tilde{\mathbf{K}}_{\mathrm{sub}} \mathcal{A}_{2, \Gamma}=\sum_{i=1}^{3}\left(\tilde{\mathbf{K}} \mathcal{A}_{1, \gamma_{i}}\right) \cdot \mathcal{A}_{1, \Gamma / \gamma_{i}}=\sum_{i=1}^{3} \int \mathrm{d} \bar{q}_{i} \delta \mathcal{R}_{1, \gamma_{i}}^{\alpha_{i}}\left(q_{i}\right) \frac{\mathcal{N}_{\alpha_{i}}^{(i)}\left(q_{i}\right)}{\mathcal{D}^{(i)}\left(\bar{q}_{i}\right)}
$$

For what concerns the subtraction of local divergences, up to negligible terms of $\mathcal{O}(\varepsilon)$ the $\mathbf{K}_{\text {loc }}$ operator in (5.23) is equivalent to its $D$-dimensional version, i.e.

$$
\mathbf{K}_{\text {loc }} \overline{\mathcal{A}}_{2, \Gamma}=\mathbf{K}_{\text {loc }} \mathcal{A}_{2, \Gamma}=-\delta Z_{2, \Gamma}
$$

where $\delta Z_{2, \Gamma}$ is the usual $\overline{\mathrm{MS}}$ two-loop counterterm. The remaining $\tilde{\mathbf{K}}_{\text {loc }}$ operator describes the $\tilde{\mathcal{N}}$-contributions stemming from local two-loop divergences and is implicitly defined through (5.23) as

$$
\tilde{\mathbf{K}}_{\mathrm{loc}} \mathcal{A}_{2, \Gamma}=\left(1-\mathbf{K}_{\mathrm{sub}}\right) \overline{\mathcal{A}}_{2, \Gamma}-\left(1-\mathbf{K}_{\mathrm{sub}}+\tilde{\mathbf{K}}_{\mathrm{sub}}\right) \mathcal{A}_{2, \Gamma}
$$

As demonstrated in the next section, such $\tilde{\mathcal{N}}$-contributions can be reconstructed through process-independent counterterms,

$$
\tilde{\mathbf{K}}_{\mathrm{loc}} \mathcal{A}_{2, \Gamma}=\delta \mathcal{R}_{2, \Gamma}
$$

which can be computed once and for all in terms of tadpole integrals. This implies that the $\delta \mathcal{R}_{2, \Gamma}$ counterterms are polynomials in the external momenta and can be described at the level of the Lagrangian in terms of local operators. 
The master formula (5.23) is equivalent to (1.5) and can be written more explicitly in terms of loop integrals as

$$
\begin{aligned}
\mathbf{R} \overline{\mathcal{A}}_{2, \Gamma}= & \int \mathrm{d} \bar{q}_{1} \int \mathrm{d} \bar{q}_{2} \frac{\mathcal{N}\left(q_{1}, q_{2}\right)}{\mathcal{D}^{(1)}\left(\bar{q}_{1}\right) \mathcal{D}^{(2)}\left(\bar{q}_{2}\right) \mathcal{D}^{(3)}\left(-\bar{q}_{1}-\bar{q}_{2}\right)} \\
& +\sum_{i=1}^{3} \int \mathrm{d} \bar{q}_{i}\left[\delta Z_{1, \gamma_{i}}^{\alpha_{i}}\left(q_{i}\right)+\delta \tilde{Z}_{1, \gamma_{i}}^{\alpha_{i}}\left(\tilde{q}_{i}\right)+\delta \mathcal{R}_{1, \gamma_{i}}^{\alpha_{i}}\left(q_{i}\right)\right] \frac{\mathcal{N}_{\alpha_{i}}^{(i)}\left(q_{i}\right)}{\mathcal{D}^{(i)}\left(\bar{q}_{i}\right)}+\left(\delta Z_{2, \Gamma}+\delta \mathcal{R}_{2, \Gamma}\right) .
\end{aligned}
$$

Note that the numerator of the two-loop integral on the r.h.s. is strictly four-dimensional, while the presence of $1 / \varepsilon$ and $\tilde{q}^{2} / \varepsilon$ poles in $\delta Z_{1, \gamma_{i}}$ and $\delta \tilde{Z}_{1, \gamma_{i}}$ requires the evaluation of one-loop integrals of type

$$
T_{N, s}^{\mu_{1} \cdots \mu_{r}}=\int \mathrm{d} \bar{q}_{1} \frac{\left(\tilde{q}^{2}\right)^{s} q_{1}^{\mu_{1}} \cdots q_{1}^{\mu_{r}}}{D_{0}\left(\bar{q}_{1}\right) \cdots D_{N-1}\left(\bar{q}_{1}\right)}
$$

up to $\mathcal{O}\left(\varepsilon^{1}\right)$, where the $q_{1}^{\mu_{i}}$ loop momenta in the numerator are four-dimensional, while the additional factor $\tilde{q}^{2}$ has power $s=0$ or 1 .

Explicit results for all relevant UV and rational counterterms in QED are presented in section 6, and the structure of the above master formula for a two-loop QED diagram is illustrated in figure 5.

\subsection{Proof and recipe for the calculation of rational terms}

As pointed out in the previous section, the master formula (5.23) should be regarded as implicit definition of the $\tilde{\mathbf{K}}_{\text {loc }}$ operator, which is explicitly defined in (5.27). By construction $\tilde{\mathbf{K}}_{\text {loc }}$ embodies the two-loop $\tilde{\mathcal{N}}$-contributions that remain after subtraction of all UV divergences and of the rational parts stemming from one-loop subdivergences. In the following we demonstrate that such $\tilde{\mathcal{N}}$-contributions can be reduced to process-independent local counterterms $\delta \mathcal{R}_{2, \Gamma}$ as anticipated in (5.28). The proof consists of two parts, which deal with diagrams with $X(\Gamma)<0$ and $X(\Gamma) \geq 0$, respectively. We also provide an explicit recipe to calculate the two-loop rational counterterms $\delta \mathcal{R}_{2, \Gamma}$ by means of tadpole integrals.

\subsubsection{Diagrams with $X(\Gamma)<0$}

We first consider generic two-loop diagrams $\Gamma$ with $X(\Gamma)<0$. This implies that $\Gamma$ is free from local divergences, i.e.

$$
\mathbf{K}_{\mathrm{loc}} \overline{\mathcal{A}}_{2, \Gamma}=0
$$

Thus only subdivergences need to be renormalised, i.e.

$$
\mathbf{R} \overline{\mathcal{A}}_{2, \Gamma}=\overline{\mathcal{A}}_{2, \Gamma}-\mathbf{K}_{\mathrm{sub}} \overline{\mathcal{A}}_{2, \Gamma} .
$$


Since for $X(\Gamma)<0$ at most one subdiagram can be UV divergent (see section 5.2), using (5.19) and (5.8), we can write ${ }^{12}$

$$
\mathbf{R} \overline{\mathcal{A}}_{2, \Gamma}=\overline{\mathcal{A}}_{2, \Gamma}-\left(\mathbf{K} \overline{\mathcal{A}}_{1, \gamma_{i}}\right) \cdot \overline{\mathcal{A}}_{1, \Gamma / \gamma_{i}}=\left(\overline{\mathcal{A}}_{1, \gamma_{i}}-\mathbf{K} \overline{\mathcal{A}}_{1, \gamma_{i}}\right) \cdot \overline{\mathcal{A}}_{1, \Gamma / \gamma_{i}}
$$

where we assume that the subdiagram $\gamma_{i}$ can be divergent or non-divergent, in which case $\mathbf{K} \overline{\mathcal{A}}_{1, \gamma_{i}}=0$, while the two remaining subdiagrams are free from divergences. The two factors on the r.h.s. of (5.36) can be related to corresponding four-dimensional quantities using

$$
\overline{\mathcal{A}}_{1, \Gamma / \gamma_{i}}=\mathcal{A}_{1, \Gamma / \gamma_{i}}+\mathcal{O}(\varepsilon),
$$

and the identity (4.25) for the $\gamma_{i}$ subdiagram, which corresponds to

$$
\overline{\mathcal{A}}_{1, \gamma_{i}}-\mathbf{K} \overline{\mathcal{A}}_{1, \gamma_{i}}=\mathcal{A}_{1, \gamma_{i}}-\mathbf{K} \mathcal{A}_{1, \gamma_{i}}+\tilde{\mathbf{K}} \mathcal{A}_{1, \gamma_{i}}+\mathcal{O}(\varepsilon),
$$

where the dependence on the loop momentum $q_{i}$ and the connecting multi-index $\alpha_{i}$ is kept implicit, and contributions of order $\tilde{q}_{i}^{2} / \varepsilon$ are consistently taken into account through the $\mathbf{K}$ operator as detailed in (4.11)-(4.14). The identities (5.37)-(5.38) can be directly applied on the r.h.s. of (5.36) neglecting all terms of $\mathcal{O}(\varepsilon)$ since the renormalised subdiagram $\gamma_{i}$ and its complement $\Gamma / \gamma_{i}$ are both free from UV singularities. This results into

$$
\mathbf{R} \overline{\mathcal{A}}_{2, \Gamma}=\left(\mathcal{A}_{1, \gamma_{i}}-\mathbf{K} \mathcal{A}_{1, \gamma_{i}}+\tilde{\mathbf{K}} \mathcal{A}_{1, \gamma_{i}}\right) \cdot \mathcal{A}_{1, \Gamma / \gamma_{i}},
$$

which can be rewritten in terms of the subtraction operators defined in (5.24)-(5.25) as

$$
\mathbf{R} \overline{\mathcal{A}}_{2, \Gamma}=\mathcal{A}_{2, \Gamma}+\left(\tilde{\mathbf{K}}_{\mathrm{sub}}-\mathbf{K}_{\text {sub }}\right) \mathcal{A}_{2, \Gamma} .
$$

This equation demonstrates the validity of the master formula (5.23) for the case $X(\Gamma)<0$ and shows that in this case neither local divergences nor $\tilde{\mathcal{N}}$-contributions of type (5.27)(5.28) occur, i.e.

$$
X(\Gamma)<0 \quad \Rightarrow \quad \mathbf{K}_{\mathrm{loc}} \mathcal{A}_{2, \Gamma}=\delta Z_{2, \Gamma}=0 \quad \text { and } \quad \tilde{\mathbf{K}}_{\mathrm{loc}} \mathcal{A}_{2, \Gamma}=\delta \mathcal{R}_{2, \Gamma}=0
$$

${ }^{12}$ The following identity can be written more explicitly as

$$
\begin{aligned}
\mathbf{R} \overline{\mathcal{A}}_{2, \Gamma} & =\int \mathrm{d} \bar{q}_{i} \int \mathrm{d} \bar{q}_{j} \frac{\overline{\mathcal{N}}\left(\bar{q}_{i}, \bar{q}_{j}\right)}{\mathcal{D}^{(i)}\left(\bar{q}_{i}\right) \mathcal{D}^{(j)}\left(\bar{q}_{j}\right) \mathcal{D}^{(k)}\left(\bar{q}_{k}\right)}+\int \mathrm{d} \bar{q}_{i} \delta Z_{1, \gamma_{i}}^{\bar{\alpha}_{i}}\left(\bar{q}_{i}\right) \frac{\overline{\mathcal{N}}_{\bar{\alpha}_{i}}^{(i)}\left(\bar{q}_{i}\right)}{\mathcal{D}^{(i)}\left(\bar{q}_{i}\right)} \\
& =\int \mathrm{d} \bar{q}_{i}\left[\int \mathrm{d} \bar{q}_{j} \frac{\bar{\Gamma}_{\bar{\alpha}}^{\bar{\alpha}_{i} \bar{\alpha}_{j} \bar{\alpha}_{k}}\left(\bar{q}_{i}, \bar{q}_{j}, \bar{q}_{k}\right) \overline{\mathcal{N}}_{\bar{\alpha}_{j}}^{(j)}\left(\bar{q}_{j}\right) \overline{\mathcal{N}}_{\bar{\alpha}_{k}}^{(k)}\left(\bar{q}_{k}\right)}{\mathcal{D}^{(j)}\left(\bar{q}_{j}\right) \mathcal{D}^{(k)}\left(\bar{q}_{k}\right)}+\delta Z_{1, \gamma_{i}}^{\bar{\alpha}_{i}}\left(\bar{q}_{i}\right)\right] \frac{\overline{\mathcal{N}}_{\bar{\alpha}_{i}}^{(i)}\left(\bar{q}_{i}\right)}{\mathcal{D}^{(i)}\left(\bar{q}_{i}\right)},
\end{aligned}
$$

where the integral representations in (5.7)-(5.8) and (5.19) are used with $\bar{q}_{k}=-\bar{q}_{i}-\bar{q}_{j}$, and $i \mid j k$ is a partition of 123. In the above integral representation, the identities (5.37) and (5.38) correspond, respectively, to

$$
\frac{\overline{\mathcal{N}}_{\bar{\alpha}_{i}}^{(i)}\left(\bar{q}_{i}\right)}{\mathcal{D}^{(i)}\left(\bar{q}_{i}\right)}=\frac{\mathcal{N}_{\alpha_{i}}^{(i)}\left(q_{i}\right)}{\mathcal{D}^{(i)}\left(\bar{q}_{i}\right)}+\mathcal{O}(\varepsilon),
$$

and

$$
[\ldots]=\int \mathrm{d} \bar{q}_{j} \frac{\Gamma^{\alpha_{i} \alpha_{j} \alpha_{k}}\left(q_{i}, q_{j}, q_{k}\right) \mathcal{N}_{\alpha_{j}}^{(j)}\left(q_{j}\right) \mathcal{N}_{\alpha_{k}}^{(k)}\left(q_{k}\right)}{\mathcal{D}^{(j)}\left(\bar{q}_{j}\right) \mathcal{D}^{(k)}\left(\bar{q}_{k}\right)}+\delta Z_{1, \gamma_{i}}^{\alpha_{i}}\left(q_{i}\right)+\delta \tilde{Z}_{1, \gamma_{i}}^{\alpha_{i}}\left(\tilde{q}_{i}\right)+\delta \mathcal{R}_{1, \gamma_{i}}^{\alpha_{i}}\left(q_{i}\right)+\mathcal{O}(\varepsilon),
$$

where [...] refers to the term between square brackets in (5.33). Note that on the r.h.s. of (5.34) and (5.35) the loop numerators of $\Gamma / \gamma_{i}$ and $\gamma_{i}$, including the connecting multi-index $\alpha_{i}$, are projected to four dimensions. 


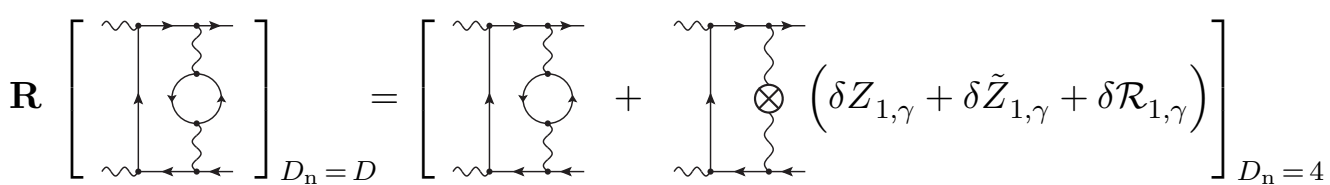

Figure 6. Graphical representation of the master formula (5.23), see also (1.5), for a two-loop QED diagram where $X(\Gamma)<0$ and thus $\delta Z_{2, \gamma}=\delta \mathcal{R}_{2, \gamma}=0$.

Thus the genuine two-loop $\tilde{\mathcal{N}}$-terms $(5.27)-(5.28)$ occur only in the finite set of diagrams that involve a local divergence. Note that (5.40) and (5.41) hold irrespectively of the presence of subdivergences, i.e. also for finite two-loop diagrams. Moreover, due to the linearity of the various $\mathbf{K}$ operators, the above identities are applicable to full diagrams, combinations of diagrams, or single pieces of individual diagrams.

A schematic representation of equation (5.40) for the case of a two-loop QED diagram with $X(\Gamma)<0$ and a subdivergence is shown in figure 6 .

\subsubsection{Diagrams with $X(\Gamma) \geq 0$}

In the following we consider two-loop diagrams $\Gamma$ with $X(\Gamma) \geq 0$, and we prove that $\tilde{\mathbf{K}}_{\text {loc }} \mathcal{A}_{2, \Gamma}$ can be cast in the form of tadpole integrals and corresponds to a local counterterm. As detailed below, the proof is based on the splitting of $\Gamma$ into two parts,

$$
\overline{\mathcal{A}}_{2, \Gamma}=\overline{\mathcal{A}}_{2, \Gamma_{\text {tad }}}+\overline{\mathcal{A}}_{2, \Gamma_{\text {rem }}}
$$

where $\Gamma_{\text {tad }}$ embodies the entire globally divergent part of $\Gamma$ in the form of pure tadpole integrals, while $\Gamma_{\text {rem }}$ is not globally divergent, i.e.

$$
X\left(\Gamma_{\text {tad }}\right)=X(\Gamma) \geq 0 \quad \text { and } \quad X\left(\Gamma_{\text {rem }}\right)<0 .
$$

This allows us to apply (5.41) to $\Gamma_{\text {rem }}$ and to conclude that

$$
\tilde{\mathbf{K}}_{\mathrm{loc}} \mathcal{A}_{2, \Gamma_{\text {rem }}}=0 \quad \text { and } \quad \tilde{\mathbf{K}}_{\mathrm{loc}} \mathcal{A}_{2, \Gamma}=\tilde{\mathbf{K}}_{\mathrm{loc}} \mathcal{A}_{2, \Gamma_{\text {tad }}}
$$

where the second relation follows form the linearity of the $\tilde{\mathbf{K}}_{\text {loc }}$ operator.

The splitting (5.42) is implemented by means of the tadpole decomposition introduced in section 3.2. Specifically, along each chain $\mathcal{C}_{i}$ of the two-loop diagram we apply an exact decomposition,

$$
\mathbf{S}_{X_{i}}^{(i)}+\mathbf{F}_{X_{i}}^{(i)}=1
$$

where the operators $\mathbf{S}_{X_{i}}^{(i)}$ and $\mathbf{F}_{X_{i}}^{(i)}$ act exclusively on the denominators depending on the loop momentum $\bar{q}_{i}$ and are defined similarly as in (3.19)-(3.23). In particular, the $\mathbf{S}_{X_{i}}^{(i)}$ operator generates the tadpole expansion

$$
\mathbf{S}_{X_{i}}^{(i)}\left(\frac{1}{\mathcal{D}^{(i)}\left(\bar{q}_{i}\right)}\right)=\sum_{\sigma=0}^{X_{i}} \frac{\Delta_{i}^{(\sigma)}\left(\bar{q}_{i}\right)}{\left(\bar{q}_{i}^{2}-M^{2}\right)^{N_{i}+\sigma}}
$$


where

$$
\Delta_{i}^{(\sigma)}\left(\bar{q}_{i}\right)=\left.\sum_{\sigma_{0}=0}^{\sigma} \ldots \sum_{\sigma_{N_{i}-1}=0}^{\sigma} \prod_{a=0}^{N_{i}-1}\left[\Delta_{i a}\left(\bar{q}_{i}\right)\right]^{\sigma_{a}}\right|_{\sigma_{0}+\cdots+\sigma_{N_{i}-1}=\sigma}
$$

with

$$
\Delta_{i a}\left(\bar{q}_{i}\right)=-p_{i a}^{2}-2 \bar{q}_{i} \cdot p_{i a}+m_{i a}^{2}-M^{2} .
$$

In practice $\mathbf{S}_{X_{i}}^{(i)}$ turns all propagators along the chain $\mathcal{C}_{i}$ into tadpoles including subleading UV contributions up to a certain relative order $1 / q_{i}^{X_{i}}$, while $\mathbf{F}_{X_{i}}^{(i)}$ collects all remnant terms, which are suppressed by order $1 / q_{i}^{X_{i}+1}$ or higher. Therefore, each $\mathbf{F}_{X_{i}}^{(i)}$ operator reduces the degree of divergence of all (sub)diagrams that involve the chain $\mathcal{C}_{i}$ by $X_{i}+1$. More explicitly, for the global degree of divergence

$$
X\left(\mathbf{F}_{X_{i}}^{(i)} \Gamma\right) \leq X(\Gamma)-\left(X_{i}+1\right)
$$

and for the degree of divergence of the subdiagrams involving the chains $\mathcal{C}_{i} \mathcal{C}_{j}$ and $\mathcal{C}_{i} \mathcal{C}_{k}$,

$$
X_{i l}\left(\mathbf{F}_{X_{i}}^{(i)} \Gamma\right) \leq X_{i l}(\Gamma)-\left(X_{i}+1\right) \quad \text { for } \quad l=j, k
$$

where $i \mid j k$ is a partition of 123 , while $X_{i k}(\Gamma)=X\left(\gamma_{j}\right)$ and $X_{i j}(\Gamma)=X\left(\gamma_{k}\right)$ are defined in (5.11). Based on (5.49)-(5.50) the order of the tadpole decompositions along the various chains is chosen as

$$
X_{i}=X_{i}(\Gamma)=\max \left\{X(\Gamma), X_{i j}(\Gamma), X_{i k}(\Gamma)\right\}
$$

which guarantees that

$$
X\left(\mathbf{F}_{X_{i}}^{(i)} \Gamma\right)<0
$$

and

$$
X_{i j}\left(\mathbf{F}_{X_{i}}^{(i)} \Gamma\right)<0, \quad X_{i k}\left(\mathbf{F}_{X_{i}}^{(i)} \Gamma\right)<0 .
$$

Thus, the remnant part $\mathbf{F}_{X_{i}}^{(i)} \Gamma$ that results from the decomposition (5.45) of a single chain $\mathcal{C}_{i}$ is completely free from global two-loop divergences and contains only $\mathrm{UV}$ divergences stemming from the subdiagram that does not involve the chain $\mathcal{C}_{i}$, i.e. the $\gamma_{i}$ subdiagram. Vice versa, the tadpole part $\mathbf{S}_{X_{i}}^{(i)} \Gamma$ contains the entire globally divergent part of $\Gamma$ as well as the UV divergences of the subdiagrams $\gamma_{j}$ and $\gamma_{k}$, which involve the chains $\mathcal{C}_{i} \mathcal{C}_{j}$ and $\mathcal{C}_{i} \mathcal{C}_{k}$, respectively.

The decomposition of all three chains can be expressed as

$$
\overline{\mathcal{A}}_{2, \Gamma}=\left(\mathbf{S}_{X_{1}}^{(1)}+\mathbf{F}_{X_{1}}^{(1)}\right)\left(\mathbf{S}_{X_{2}}^{(2)}+\mathbf{F}_{X_{2}}^{(2)}\right)\left(\mathbf{S}_{X_{3}}^{(3)}+\mathbf{F}_{X_{3}}^{(3)}\right) \overline{\mathcal{A}}_{2, \Gamma},
$$

or, more explicitly,

$$
\overline{\mathcal{A}}_{2, \Gamma}=\int \mathrm{d} \bar{q}_{1} \int \mathrm{d} \bar{q}_{2} \overline{\mathcal{N}}\left(\bar{q}_{1}, \bar{q}_{2}\right) \prod_{i=1}^{3}\left[\left(\mathbf{S}_{X_{i}}^{(i)}+\mathbf{F}_{X_{i}}^{(i)}\right) \frac{1}{\mathcal{D}^{(i)}\left(\bar{q}_{i}\right)}\right]_{q_{3}=-q_{1}-q_{2}} .
$$


Expanding the r.h.s. of (5.54) results into eight different combinations of $\mathbf{S}$ and $\mathbf{F}$ operators, which can be grouped into two contributions that correspond to the tadpole and remnant parts introduced in (5.42),

$$
\begin{aligned}
& \overline{\mathcal{A}}_{2, \Gamma_{\text {tad }}}=\mathbf{S}_{X_{1}}^{(1)} \mathbf{S}_{X_{2}}^{(2)} \mathbf{S}_{X_{3}}^{(3)} \overline{\mathcal{A}}_{2, \Gamma} \\
& \overline{\mathcal{A}}_{2, \Gamma_{\mathrm{rem}}}=\mathbf{F}_{X_{1}}^{(1)} \mathbf{F}_{X_{2}}^{(2)} \mathbf{F}_{X_{3}}^{(3)} \overline{\mathcal{A}}_{2, \Gamma}+\sum_{i=1}^{3} \mathbf{S}_{X_{i}}^{(i)} \mathbf{F}_{X_{j}}^{(j)} \mathbf{F}_{X_{k}}^{(k)} \overline{\mathcal{A}}_{2, \Gamma}+\sum_{i=1}^{3} \mathbf{F}_{X_{i}}^{(i)} \mathbf{S}_{X_{j}}^{(j)} \mathbf{S}_{X_{k}}^{(k)} \overline{\mathcal{A}}_{2, \Gamma} .
\end{aligned}
$$

In (5.56) all three chains are replaced by their tadpole expansion, i.e. $\Gamma_{\text {tad }}$ consists only of tadpole integrals. In contrast, the remaining seven combinations in (5.57) involve at least one $\mathbf{F}$ operator. Thus (5.52) guarantees that the $\Gamma_{\text {rem }}$ part is free form global divergences and does not contribute to $\tilde{\mathbf{K}}_{\text {loc }} \mathcal{A}_{2, \Gamma}$, as anticipated in (5.43)-(5.44).

Combining (5.44) with (5.27) we obtain

$$
\tilde{\mathbf{K}}_{\text {loc }} \mathcal{A}_{2, \Gamma}=\left(1-\mathbf{K}_{\text {sub }}\right) \overline{\mathcal{A}}_{2, \Gamma_{\text {tad }}}-\left(1-\mathbf{K}_{\text {sub }}+\tilde{\mathbf{K}}_{\text {sub }}\right) \mathcal{A}_{2, \Gamma_{\text {tad }}} .
$$

Here the interplay between the tadpole expansion (5.56) and the $\mathbf{K}_{\text {sub }}$ operators results into tadpole terms like

$$
\begin{aligned}
\mathbf{K}_{\text {sub }} \overline{\mathcal{A}}_{2, \Gamma_{\text {tad }}} & =\sum_{i=1}^{3}\left(\mathbf{K} \overline{\mathcal{A}}_{1, \gamma_{\mathrm{tad}, i}}\right) \cdot \overline{\mathcal{A}}_{2, \Gamma_{\mathrm{tad}} / \gamma_{\mathrm{tad}, i}}=\sum_{i=1}^{3}\left(\mathbf{K} \mathbf{S}_{X_{j}}^{(j)} \mathbf{S}_{X_{k}}^{(k)} \overline{\mathcal{A}}_{1, \gamma_{i}}\right) \cdot\left(\mathbf{S}_{X_{i}}^{(i)} \overline{\mathcal{A}}_{2, \Gamma / \gamma_{i}}\right) \\
& =\sum_{i=1}^{3}\left(\mathbf{K} \overline{\mathcal{A}}_{1, \gamma_{i}}\right) \cdot\left(\mathbf{S}_{X_{i}}^{(i)} \overline{\mathcal{A}}_{2, \Gamma / \gamma_{i}}\right)
\end{aligned}
$$

where the second identity is guaranteed by the fact that the $\mathbf{S}$ operators in (5.56) factorise, in the sense that each $\mathbf{S}_{X_{i}}^{(i)}$ acts only on the corresponding chain $\mathcal{C}_{i}$. The third identity is based on (5.53), which guarantees that the tadpole expansions $\mathbf{S}_{X_{j}}^{(j)}$ and $\mathbf{S}_{X_{k}}^{(k)}$ capture the full UV divergent part of the subdiagram $\gamma_{i}$ containing the chains $\mathcal{C}_{j}$ and $\mathcal{C}_{k}$. Using similar identities for the terms $\mathbf{K}_{\text {sub }} \mathcal{A}_{2, \Gamma_{\text {tad }}}$ and $\tilde{\mathbf{K}}_{\text {sub }} \mathcal{A}_{2, \Gamma_{\text {tad }}}$ in (5.58) we arrive at

$$
\begin{aligned}
\tilde{\mathbf{K}}_{\mathrm{loc}} \mathcal{A}_{2, \Gamma}= & {\left[\prod_{i=1}^{3} \mathbf{S}_{X_{i}}^{(i)} \overline{\mathcal{A}}_{2, \Gamma}-\sum_{i=1}^{3}\left(\mathbf{K} \overline{\mathcal{A}}_{1, \gamma_{i}}\right) \cdot\left(\mathbf{S}_{X_{i}}^{(i)} \overline{\mathcal{A}}_{2, \Gamma / \gamma_{i}}\right)\right] } \\
& -\left[\prod_{i=1}^{3} \mathbf{S}_{X_{i}}^{(i)} \mathcal{A}_{2, \Gamma}-\sum_{i=1}^{3}\left(\mathbf{K} \mathcal{A}_{1, \gamma_{i}}-\tilde{\mathbf{K}} \mathcal{A}_{1, \gamma_{i}}\right) \cdot\left(\mathbf{S}_{X_{i}}^{(i)} \mathcal{A}_{2, \Gamma / \gamma_{i}}\right)\right],
\end{aligned}
$$

which can be written more explicitly as

$$
\begin{aligned}
\delta \mathcal{R}_{2, \Gamma}= & \int \mathrm{d} \bar{q}_{1} \int \mathrm{d} \bar{q}_{2}\left[\overline{\mathcal{N}}\left(\bar{q}_{1}, \bar{q}_{2}\right)-\mathcal{N}\left(q_{1}, q_{2}\right)\right]\left[\prod_{i=1}^{3} \mathbf{S}_{X_{i}}^{(i)} \frac{1}{\mathcal{D}^{(i)}\left(\bar{q}_{i}\right)}\right]_{q_{3}=-q_{1}-q_{2}} \\
& +\sum_{i=1}^{3} \int \mathrm{d} \bar{q}_{i}\left[\delta Z_{1, \gamma_{i}}^{\bar{\alpha}_{i}}\left(\bar{q}_{i}\right) \overline{\mathcal{N}}_{\bar{\alpha}_{i}}^{(i)}\left(\bar{q}_{i}\right)-\left(\delta Z_{1, \gamma_{i}}^{\alpha_{i}}\left(q_{i}\right)+\delta \tilde{Z}_{1, \gamma_{i}}^{\alpha_{i}}\left(\tilde{q}_{i}\right)+\delta \mathcal{R}_{1, \gamma_{i}}^{\alpha_{i}}\left(q_{i}\right)\right) \mathcal{N}_{\alpha_{i}}^{(i)}\left(q_{i}\right)\right] \\
& \times \mathbf{S}_{X_{i}}^{(i)}\left(\frac{1}{\mathcal{D}^{(i)}\left(\bar{q}_{i}\right)}\right) .
\end{aligned}
$$




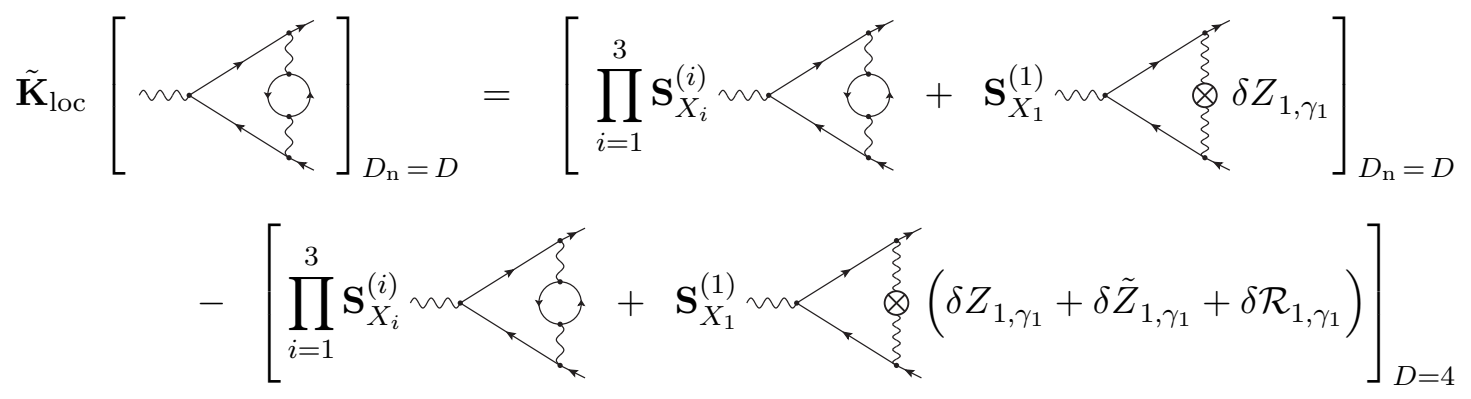

Figure 7. Graphical representation of the master formula (5.60) for the derivation of two-loop rational counterterms for the case of a globally divergent two-loop diagram with a single divergent subdiagram $\gamma_{1}$. The $\mathbf{S}_{X_{i}}^{(i)}$ operators perform tadpole expansions along the corresponding chains $\mathcal{C}_{i}$, and the subtracted one-loop contributions involve a single tadpole expansion along the chain $\mathcal{C}_{1}$ associated with the complement $\Gamma / \gamma_{1}$ of the divergent subdiagram.

The identities (5.60)-(5.61) represent the master formulas for the calculation of the $\delta \mathcal{R}_{2, \Gamma}$ counterterms in terms of tadpole integrals. Moreover, the structure of these formulas provides insights into the general properties of the $\delta \mathcal{R}_{2, \Gamma}$ counterterms. In particular, from the form of (5.61) and (5.46)-(5.48) it is evident that such counterterms are polynomials in the external momenta $\left\{p_{i a}\right\}$ and internal masses $\left\{m_{i a}\right\}$. With other words, the $\delta \mathcal{R}_{2, \Gamma}$ counterterms correspond to local operators at the Lagrangian level, and at the level of scattering amplitudes they result into rational functions of the kinematic invariants.

The various tadpole expansions in (5.61) give rise to terms depending on the auxiliary mass scale $M^{2}$. However, this dependence cancels in $\delta \mathcal{R}_{2, \Gamma}$. This is guaranteed by the fact that the tadpole decomposition (5.54) is exact, and thus independent of $M^{2}$, while the contribution of the amputated remnant (5.57) to $\delta \mathcal{R}_{2}$ vanishes. This implies that $\delta \mathcal{R}_{2}$ counterterms are also independent of the renormalisation scale $\mu$, since such dependence could arise only in the form of logarithms of $M^{2} / \mu^{2}$ in the tadpole integrals ${ }^{13}$ on the r.h.s. of (5.61).

The master formula (5.61) can be optimised in various ways. For instance, the number of tadpole integrals to be computed can be significantly reduced by applying a strict power counting in $1 / q_{i}$ such that all terms of relative order higher than $1 / q_{i}^{X_{i}}$ are shifted from the $\mathbf{S}_{X_{i}}^{(i)}$ operators to the $\mathbf{F}_{X_{i}}^{(i)}$ remnants. Moreover, the fact that the resulting $\delta \mathcal{R}_{2, \Gamma}$ terms are homogenous polynomials of degree $X(\Gamma)$ in $\left\{p_{i a}, m_{i a}\right\}$ allows one to discard all terms of different order at the integrand level. The results presented in section 6 have been obtained by selecting the terms of order $X(\Gamma)$ in $\left\{p_{i a}, m_{i a}\right\}$ and discarding also all irrelevant $M^{2}$-dependent terms in the loop numerators. This can be achieved by omitting all $M^{2}$-contributions in (5.47) and then reconstructing the correct $M^{2}$-dependence through auxiliary one-loop counterterms along the lines of $[16,17,25]$. Results obtained in this way have been validated against a naive implementation of the tadpole expansions as described in (5.46)-(5.47). More details on the implementation of the tadpole expansion and its optimisations will be discussed in a forthcoming paper.

\footnotetext{
${ }^{13}$ In general the rational terms depend on the ratio between the regularisation and renormalisation scales. However these two scales are set equal to each other in this paper.
} 
The master formula (5.60)-(5.61) for the calculation of two-loop rational counterterms is illustrated in figure 7 for the case of a two-loop QED diagram with a single subdivergence.

\section{Two-loop rational terms in QED}

As a first application of the method introduced in section 5 we have derived the full set of two-loop rational terms $\delta \mathcal{R}_{2}$ in QED in the $\overline{\mathrm{MS}}$ scheme. To this end we have applied the master formula (5.61) to the full set of globally divergent Feynman diagrams for the various 2-, 3-, and 4-point 1PI functions in QED. For convenience, let us summarise the various ingredients that are required for the implementation of (5.61). As defined in (5.1)-(5.6), the function $\overline{\mathcal{N}}\left(\bar{q}_{1}, \bar{q}_{2}\right)$ is the numerator of the two-loop diagram at hand in $D$ dimensions, $\mathcal{N}\left(q_{1}, q_{2}\right)$ represents its four-dimensional projection, and $\mathcal{D}^{(i)}\left(\bar{q}_{i}\right)$ are the associated chains of denominators. The functions $\overline{\mathcal{N}}_{\bar{\alpha}_{i}}^{(i)}\left(\bar{q}_{i}\right)$ and $\mathcal{N}_{\alpha_{i}}^{(i)}\left(q_{i}\right)$ represent, respectively, the $D$ - and four-dimensional numerator of the complement $\Gamma / \gamma_{i}$ of the subdiagram $\gamma_{i}$. Their relation with the numerator of the two-loop diagram $\Gamma$ is specified in (5.7)-(5.8). The required oneloop counterterms and rational terms associated with the $\gamma_{i}$ subdiagrams, i.e. $\delta Z_{1, \gamma_{i}}^{\alpha_{i}}\left(q_{i}\right)$, $\delta \tilde{Z}_{1, \gamma_{i}}^{\alpha_{i}}\left(\tilde{q}_{i}\right)$, and $\delta \mathcal{R}_{1, \gamma_{i}}^{\alpha_{i}}\left(q_{i}\right)$, can be found below and in appendix A. Finally, the tadpole expansion operators $\mathbf{S}_{X_{i}}^{(i)}$ are defined in (5.46)-(5.48) (see also section 3.2), and the expansion order $X_{i}$ is dictated by (5.11)-(5.12) and (5.51). After the $\mathbf{S}_{X_{i}}^{(i)}$ expansions one is left with the evaluation of massive tadpole integrals.

In practice, the master formula (5.61) and all relevant building blocks have been implemented in the GeFicom [26] framework, which is based on QGRAF [27], Q2E and ExP $[28,29]$ for the generation and topology identification of Feynman diagrams, and implements the relevant algebraic manipulations, one-loop insertions and tadpole decompositions in FORM [30, 31], while massive tadpole integrals are computed with MATAD [32].

We consider the QED Lagrangian

$$
\mathcal{L}_{\mathrm{QED}}=\bar{\psi}\left(\mathrm{i} \gamma^{\mu} D_{\mu}-m\right) \psi-\frac{1}{4} F_{\mu \nu} F^{\mu \nu}-\frac{1}{2 \lambda}\left(\partial^{\mu} A_{\mu}\right)^{2},
$$

with $D_{\mu}=\partial_{\mu}-i e A_{\mu}$ and a generic gauge parameter $\lambda$. The corresponding Feynman rules are listed in appendix A together with the known one- and two-loop counterterms in the $\overline{\mathrm{MS}}$ scheme. In the following we present results for the rational terms at one and two loops in $D=4-2 \varepsilon$ dimensions. For convenience we write our results in the form

$$
\delta \mathcal{R}_{k, \gamma}^{\alpha_{1} \ldots \alpha_{N}}=\mathrm{i}\left(\frac{\alpha}{4 \pi}\right)^{k} S^{k \varepsilon} \sum_{a} \delta \hat{\mathcal{R}}_{k, \gamma}^{(a)} \mathcal{T}_{a, \gamma}^{\alpha_{1} \ldots \alpha_{N}}
$$

where $k=1,2$ is the loop order, $\alpha=e^{2} /(4 \pi), S^{\varepsilon}$ is the $\overline{\mathrm{MS}}$ normalisation factor defined in (3.28), and $\mathcal{T}_{a, \gamma}^{\alpha_{1} \ldots \alpha_{N}}$ are independent tensor structures carrying the indices $\alpha_{1} \ldots \alpha_{N}$ of the external lines of the vertex function at hand. For convenience the gauge dependence is expressed in terms of $\eta=1-\lambda$, i.e. the Feynman gauge corresponds to $\eta=0$.

The rational terms for the electron two-point function have the form

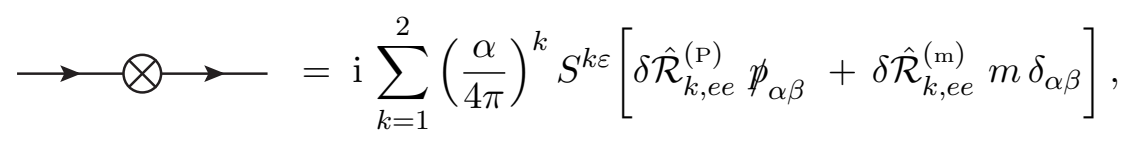


with two tensor structures, $\mathcal{T}_{\mathrm{P}, e e}^{\alpha \beta}=\not p_{\alpha \beta}, \mathcal{T}_{\mathrm{m}, e e}^{\alpha \beta}=m \delta_{\alpha \beta}$, and the coefficients

$$
\begin{array}{ll}
\delta \hat{\mathcal{R}}_{1, e e}^{(\mathrm{P})}=-1+\frac{2}{3} \eta, & \delta \hat{\mathcal{R}}_{2, e e}^{(\mathrm{P})}=\left(\frac{19}{18}-\frac{143}{72} \eta+\frac{11}{30} \eta^{2}\right) \varepsilon^{-1}+\left(\frac{247}{108}+\frac{293}{864} \eta+\frac{391}{14400} \eta^{2}\right), \\
\delta \hat{\mathcal{R}}_{1, e e}^{(\mathrm{m})}=2-\frac{1}{2} \eta, & \delta \hat{\mathcal{R}}_{2, e e}^{(\mathrm{m})}=\left(-11+\frac{41}{9} \eta-\frac{1}{4} \eta^{2}\right) \varepsilon^{-1}+\left(-\frac{5}{6}-\frac{13}{54} \eta-\frac{7}{288} \eta^{2}\right) .
\end{array}
$$

As usual the direction of the momentum $p$ in (6.3) coincides with the fermion flow.

For the photon two-point function we have

$$
\mu \sim \otimes \sim \nu=\mathrm{i} \sum_{k=1}^{2}\left(\frac{\alpha}{4 \pi}\right)^{k} S^{k \varepsilon}\left[\delta \hat{\mathcal{R}}_{k, \gamma \gamma}^{(\mathrm{P})} p^{\mu} p^{\nu}+\left(\delta \hat{\mathcal{R}}_{k, \gamma \gamma}^{(\mathrm{G})} p^{2}+\delta \tilde{Z}_{k, \gamma \gamma}^{(\mathrm{G})} \tilde{p}^{2}\right) g^{\mu \nu}\right]
$$

with two tensor structures, $\mathcal{T}_{\mathrm{P}, \gamma \gamma}^{\mu \nu}=p^{\mu} p^{\nu}, \mathcal{T}_{\mathrm{G}, \gamma \gamma}^{\mu \nu}=p^{2} g^{\mu \nu}$, and the coefficients

$$
\begin{array}{ll}
\delta \hat{\mathcal{R}}_{1, \gamma \gamma}^{(\mathrm{P})}=0, & \delta \hat{\mathcal{R}}_{2, \gamma \gamma}^{(\mathrm{P})}=\left(\frac{2}{3}+\frac{4}{9} \eta\right) \varepsilon^{-1}+\left(-\frac{71}{18}+\frac{59}{108} \eta\right), \\
\delta \hat{\mathcal{R}}_{1, \gamma \gamma}^{(\mathrm{G})}=\frac{2}{3}-4 \frac{m^{2}}{p^{2}}, & \delta \hat{\mathcal{R}}_{2, \gamma \gamma}^{(\mathrm{G})}=\left(-\frac{2}{3}-\frac{4}{9} \eta+6 \frac{m^{2}}{p^{2}}\right) \varepsilon^{-1}+\left(\frac{109}{36}-\frac{73}{216} \eta+7 \frac{m^{2}}{p^{2}}\right) .
\end{array}
$$

In addition, due to the presence of a quadratic divergence, the usual $\overline{\mathrm{MS}}$ counterterm for the photon two-point function needs to be supplemented by

$$
\delta \tilde{Z}_{1, \gamma \gamma}^{(\mathrm{G})}=\frac{2}{3} \varepsilon^{-1} .
$$

This extra term is relevant only when it is inserted in a one-loop diagram in the context of two-loop calculations, and its two-loop extension $\delta \tilde{Z}_{2, \gamma \gamma}^{(\mathrm{G})}$ is required only for calculations beyond two loops.

For the electron-photon vertex we have

$$
\mu \sim=\mathrm{i} e \gamma^{\mu} \sum_{k=1}^{2}\left(\frac{\alpha}{4 \pi}\right)^{k} S^{k \varepsilon} \delta \hat{\mathcal{R}}_{k, e e \gamma}^{(\mathrm{v})}
$$

with a single tensor structure, $\mathcal{T}_{\mathrm{v}, e e \gamma}^{\mu}=e \gamma^{\mu}$, and

$$
\delta \hat{\mathcal{R}}_{1, e e \gamma}^{(\mathrm{v})}=-2+\frac{5}{6} \eta, \quad \delta \hat{\mathcal{R}}_{2, \text { ee }}^{(\mathrm{v})}=\left(\frac{13}{9}-\frac{35}{12} \eta+\frac{29}{60} \eta^{2}\right) \varepsilon^{-1}+\left(\frac{191}{27}-\frac{17}{24} \eta+\frac{123}{1600} \eta^{2}\right) .
$$

Finally, for the quartic photon vertex we have

$$
\int_{\rho}^{\mu}=\mathrm{i} e^{2}\left(g^{\mu \nu} g^{\rho \sigma}+g^{\mu \rho} g^{\nu \sigma}+g^{\mu \sigma} g^{\nu \rho}\right) \sum_{k=1}^{2}\left(\frac{\alpha}{4 \pi}\right)^{k} S^{k \varepsilon} \delta \hat{\mathcal{R}}_{k, 4 \gamma}^{(\mathrm{s})},
$$


with a single tensor structure, $\mathcal{T}_{\mathrm{S}, 4 \gamma}^{\mu \nu \rho \sigma}=g^{\mu \nu} g^{\rho \sigma}+g^{\mu \rho} g^{\nu \sigma}+g^{\mu \sigma} g^{\nu \rho}$, and

$$
\delta \hat{\mathcal{R}}_{1,4 \gamma}^{(\mathrm{S})}=\frac{4}{3}, \quad \delta \hat{\mathcal{R}}_{2,4 \gamma}^{(\mathrm{s})}=-3+\frac{1}{2} \eta .
$$

At one loop, the rational counterterms $\delta \mathcal{R}_{1, \gamma}$ are in agreement with the results obtained in [7] for $\eta=0$, while their $\eta$-dependent parts are presented here for the first time. At two loops, the form of the rational counterterms $\delta \mathcal{R}_{2, \Gamma}$ confirms the conclusions of the general analysis of section 5 , namely that $\delta \mathcal{R}_{2, \Gamma}$ are polynomials of the external momenta and internal masses. We also note that, due to the presence of $1 / \varepsilon^{2} \mathrm{UV}$ poles at two loops, the $\delta \mathcal{R}_{2, \Gamma}$ terms contain single $1 / \varepsilon$ poles. Moreover, as expected, the $\delta \mathcal{R}_{2, \Gamma}$ counterterms are independent of the auxiliary tadpole mass $M$.

\section{$7 \quad$ Summary and conclusions}

The construction of one-loop scattering amplitudes through efficient numerical algorithms that handle the numerator of loop integrands in $D_{\mathrm{n}}=4$ dimensions turned out to be a very successful strategy for the automation of NLO calculations. When the loop numerator is restricted to four dimensions, the contributions associated with its $(D-4)$-dimensional counterpart, referred to as $\tilde{\mathcal{N}}$, need to be reconstructed with a different technique. At one loop, $\tilde{\mathcal{N}}$-contributions can be reconstructed in a very efficient way through the insertion of process-independent rational counterterms into tree amplitudes. In order to open the door to the usage of two-loop numerical algorithms in $D_{\mathrm{n}}=4$ numerator dimensions, in this paper we have presented a general analysis of rational $\tilde{\mathcal{N}}$-contributions at two loops. Such contributions can arise from the interplay of $\tilde{\mathcal{N}}$ with $1 /(D-4)$ poles of UV or IR kind, and we have focused on poles of UV kind, deferring the study of IR poles to future work.

The main result is a formula that relates generic renormalised two-loop amplitudes with loop numerators in $D_{\mathrm{n}}=D$ and $D_{\mathrm{n}}=4$ dimensions. Its structure is similar to the well-known R-operation for the subtraction of UV divergences. Renormalised two-loop amplitudes are expressed as a combination of unrenormalised two-loop amplitudes, oneloop counterterm insertions into one-loop amplitudes, and two-loop counterterm insertions into tree amplitudes. In this formula the well known $\overline{\mathrm{MS}}$ counterterms for the subtraction of UV divergences are accompanied by rational counterterms for the reconstruction of the related $\tilde{\mathcal{N}}$-contributions. In addition, the one-loop $\overline{\mathrm{MS}}$ counterterms for quadratically divergent subdiagrams need to be supplemented by extra UV counterterms proportional to $\tilde{q}^{2} /(D-4)$, where $\tilde{q}$ denotes the $(D-4)$-dimensional part of the loop momentum. The $\tilde{\mathcal{N}}$-contributions associated with one-loop subdivergences are reconstructed through insertions of the well-known one-loop rational counterterms into one-loop amplitudes, while the remaining $\tilde{\mathcal{N}}$-contributions associated with local two-loop divergences are reconstructed through the insertion of two-loop rational counterterms into tree amplitudes.

We have demonstrated that two-loop rational counterterms are process-independent polynomials of the external momenta and internal masses. They can be extracted from a finite set of superficially divergent two-loop diagrams, and for their derivation we have presented a general formula, applicable to any renormalisable theory, where the relevant 
two-loop diagrams are reduced to massive tadpole integrals with one auxiliary mass scale, of which the result is independent. As a first application we have presented the full set of two-loop rational counterterms for QED in the $R_{\xi}$-gauge.

\section{Acknowledgments}

We thank J.N. Lang for numerous discussions. H.Z. also thanks A. Primo and T. Peraro for discussions. This research was supported by the Swiss National Science Foundation (SNSF) under contract BSCGI0-157722, and the work of M.Z. was also supported through the SNSF Ambizione grant PZ00P2-179877.

\section{A Feynman rules and UV counterterms in QED}

For convenience of the reader we list the Feynman rules for the QED Lagrangian (6.1) together with the full set of $\overline{\mathrm{MS}}$ counterterms at one and two loops. Similarly as in section 6 , the results correspond to $D=4-2 \varepsilon$ dimensions and are parametrised in terms of $\eta=$ $1-\lambda$, with $\eta=0$ corresponding to the Feynman gauge. In analogy with (6.2), the UV counterterms are expressed as

$$
\delta Z_{k, \gamma_{i}}^{\alpha_{1} \ldots \alpha_{N}}=\mathrm{i}\left(\frac{\alpha}{4 \pi}\right)^{k} S^{k \varepsilon} \sum_{a} \delta \hat{Z}_{k, \gamma_{i}}^{(a)} \mathcal{T}_{a}^{\alpha_{1} \ldots \alpha_{N}}
$$

using the same tensor structures as in section 6 and the $\overline{\mathrm{MS}}$ normalisation factor (3.28). The UV counterterms were computed in the same framework as the rational terms and agree with those in the literature, which have been available for a long time [33-35].

For the electron and photon two-point functions we have

$$
\longrightarrow \otimes-\mathrm{i}\left\{(\not p-m)_{\alpha \beta}+\sum_{k=1}^{2}\left(\frac{\alpha}{4 \pi}\right)^{k} S^{k \varepsilon}\left[\delta \hat{Z}_{k, e e}^{(\mathrm{P})} \not p_{\alpha \beta}-\delta \hat{Z}_{k, e e}^{(\mathrm{m})} m \delta_{\alpha \beta}\right]\right\},
$$

with

$$
\begin{array}{ll}
\delta \hat{Z}_{1, e e}^{(\mathrm{P})}=(-1+\eta) \varepsilon^{-1}, & \delta \hat{Z}_{2, e e}^{(\mathrm{P})}=\left(\frac{1}{2}-\eta+\frac{1}{2} \eta^{2}\right) \varepsilon^{-2}+\frac{7}{4} \varepsilon^{-1}, \\
\delta \hat{Z}_{1, e e}^{(\mathrm{m})}=(-4+\eta) \varepsilon^{-1}, & \delta \hat{Z}_{2, e e}^{(\mathrm{m})}=\left(6-4 \eta+\frac{1}{2} \eta^{2}\right) \varepsilon^{-2}+\frac{8}{3} \varepsilon^{-1},
\end{array}
$$

and

$$
\mu \sim \otimes \sim \nu=-\mathrm{i}\left\{p^{2} g^{\mu \nu}+\left(\frac{1}{\lambda}-1\right) p^{\mu} p^{\nu}+\sum_{k=1}^{2}\left(\frac{\alpha}{4 \pi}\right)^{k} S^{k \varepsilon} \delta \hat{Z}_{k, \gamma \gamma}^{(\mathrm{T})}\left(p^{2} g^{\mu \nu}-p^{\mu} p^{\nu}\right)\right\},
$$

with

$$
\delta \hat{Z}_{1, \gamma \gamma}^{(\mathrm{T})}=-\frac{4}{3} \varepsilon^{-1}, \quad \delta \hat{Z}_{2, \gamma \gamma}^{(\mathrm{T})}=-2 \varepsilon^{-1}
$$


For the electron-photon vertex

$$
\mu \sim \mathrm{i} e \gamma^{\mu}\left\{1+\sum_{k=1}^{2}\left(\frac{\alpha}{4 \pi}\right)^{k} S^{k \varepsilon} \delta \hat{Z}_{k, e e \gamma}^{(\mathrm{v})}\right\},
$$

with

$$
\delta \hat{Z}_{1, e e \gamma}^{(\mathrm{v})}=(-1+\eta) \varepsilon^{-1}, \quad \delta \hat{Z}_{2, e e \gamma}^{(\mathrm{v})}=\left(\frac{1}{2}-\eta+\frac{1}{2} \eta^{2}\right) \varepsilon^{-2}+\frac{7}{4} \varepsilon^{-1} .
$$

In the context of two-loop calculations, when the one-loop counterterms $\delta Z_{1, \gamma}$ are inserted into one-loop diagrams, the associated tensor structures and their loop-momentum dependence have to be adapted to the dimensionality of the loop numerator, i.e. using $\delta Z_{1, \gamma}^{\bar{\alpha}_{1} \ldots \bar{\alpha}_{N}}\left(\bar{q}_{1}\right)$ and $\delta Z_{1, \gamma}^{\alpha_{1} \ldots \alpha_{N}}\left(q_{1}\right)$, respectively, in $D_{\mathrm{n}}=D$ and $D_{\mathrm{n}}=4$ numerator dimensions. Moreover, in the master formula (5.61) the four-dimensional $\overline{\mathrm{MS}}$ counterterm needs to be supplemented by the additional $\delta \tilde{Z}_{1, \gamma}\left(\tilde{q}_{1}\right)$ counterterm. The latter is not included in the above formulas since it can be found in section 6 .

Open Access. This article is distributed under the terms of the Creative Commons Attribution License (CC-BY 4.0), which permits any use, distribution and reproduction in any medium, provided the original author(s) and source are credited.

\section{References}

[1] G. 't Hooft and M.J.G. Veltman, Regularization and Renormalization of Gauge Fields, Nucl. Phys. B 44 (1972) 189 [INSPIRE].

[2] C. Gnendiger et al., To $d$, or not to d: recent developments and comparisons of regularization schemes, Eur. Phys. J. C 77 (2017) 471 [arXiv:1705.01827] [INSPIRE].

[3] F. Buccioni et al., OpenLoops 2, Eur. Phys. J. C 79 (2019) 866 [arXiv:1907.13071] [INSPIRE].

[4] A. Denner, J.-N. Lang and S. Uccirati, Recola2: REcursive Computation of One-Loop Amplitudes 2, Comput. Phys. Commun. 224 (2018) 346 [arXiv:1711.07388] [INSPIRE].

[5] A. van Hameren, C.G. Papadopoulos and R. Pittau, Automated one-loop calculations: A proof of concept, JHEP 09 (2009) 106 [arXiv:0903.4665] [INSPIRE].

[6] V. Hirschi, R. Frederix, S. Frixione, M.V. Garzelli, F. Maltoni and R. Pittau, Automation of one-loop QCD corrections, JHEP 05 (2011) 044 [arXiv:1103.0621] [INSPIRE].

[7] G. Ossola, C.G. Papadopoulos and R. Pittau, On the Rational Terms of the one-loop amplitudes, JHEP 05 (2008) 004 [arXiv: 0802.1876] [INSPIRE].

[8] P. Draggiotis, M.V. Garzelli, C.G. Papadopoulos and R. Pittau, Feynman Rules for the Rational Part of the QCD 1-loop amplitudes, JHEP 04 (2009) 072 [arXiv:0903.0356] [INSPIRE].

[9] M.V. Garzelli, I. Malamos and R. Pittau, Feynman rules for the rational part of the Electroweak 1-loop amplitudes, JHEP 01 (2010) 040 [Erratum ibid. 10 (2010) 097] [arXiv:0910.3130] [INSPIRE]. 
[10] R. Pittau, Primary Feynman rules to calculate the epsilon-dimensional integrand of any 1-loop amplitude, JHEP 02 (2012) 029 [arXiv:1111.4965] [INSPIRE].

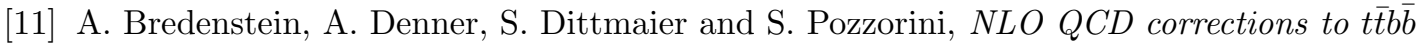
production at the LHC: 1. Quark-antiquark annihilation, JHEP 08 (2008) 108 [arXiv:0807.1248] [INSPIRE].

[12] N.N. Bogoliubov and O.S. Parasiuk, On the Multiplication of the causal function in the quantum theory of fields, Acta Math. 97 (1957) 227.

[13] K. Hepp, Proof of the Bogolyubov-Parasiuk theorem on renormalization, Commun. Math. Phys. 2 (1966) 301 [INSPIRE].

[14] W. Zimmermann, Convergence of Bogolyubov's method of renormalization in momentum space, Commun. Math. Phys. 15 (1969) 208 [INSPIRE].

[15] W.E. Caswell and A.D. Kennedy, A simple approach to renormalization theory, Phys. Rev. D 25 (1982) 392 [INSPIRE].

[16] M. Misiak and M. Münz, Two loop mixing of dimension five flavor changing operators, Phys. Lett. B 344 (1995) 308 [hep-ph/9409454] [INSPIRE].

[17] K.G. Chetyrkin, M. Misiak and M. Münz, $\beta$-functions and anomalous dimensions up to three loops, Nucl. Phys. B 518 (1998) 473 [hep-ph/9711266] [INSPIRE].

[18] B. Page and R. Pittau, Two-loop off-shell QCD amplitudes in FDR, JHEP 11 (2015) 183 [arXiv: 1506.09093] [INSPIRE].

[19] R. Pittau, A four-dimensional approach to quantum field theories, JHEP 11 (2012) 151 [arXiv: 1208.5457] [INSPIRE].

[20] F. del Aguila and R. Pittau, Recursive numerical calculus of one-loop tensor integrals, JHEP 07 (2004) 017 [hep-ph/0404120] [INSPIRE].

[21] G. Ossola, C.G. Papadopoulos and R. Pittau, Reducing full one-loop amplitudes to scalar integrals at the integrand level, Nucl. Phys. B 763 (2007) 147 [hep-ph/0609007] [INSPIRE].

[22] Z. Bern and D.A. Kosower, The computation of loop amplitudes in gauge theories, Nucl. Phys. B 379 (1992) 451 [INSPIRE].

[23] Z. Bern, A. De Freitas, L.J. Dixon and H.L. Wong, Supersymmetric regularization, two loop QCD amplitudes and coupling shifts, Phys. Rev. D 66 (2002) 085002 [hep-ph/0202271] [INSPIRE].

[24] R.A. Fazio, P. Mastrolia, E. Mirabella and W.J. Torres Bobadilla, On the Four-Dimensional Formulation of Dimensionally Regulated Amplitudes, Eur. Phys. J. C 74 (2014) 3197 [arXiv: 1404.4783] [INSPIRE].

[25] M.F. Zoller, Three-loop $\beta$-function for the Higgs self-coupling, PoS (LL2014) 014 [arXiv:1407.6608] [INSPIRE].

[26] K.G. Chetyrkin and M.F. Zoller, GEFICOM, private code.

[27] P. Nogueira, Automatic Feynman graph generation, J. Comput. Phys. 105 (1993) 279.

[28] T. Seidensticker, Automatic application of successive asymptotic expansions of Feynman diagrams, in 6th International Workshop on New Computing Techniques in Physics Research: Software Engineering, Artificial Intelligence Neural Nets, Genetic Algorithms, Symbolic Algebra, Automatic Calculation (AIHENP 99) Heraklion, Crete, Greece, April 12-16, 1999, hep-ph/9905298 [INSPIRE]. 
[29] R. Harlander, T. Seidensticker and M. Steinhauser, Complete corrections of $O\left(\alpha \alpha_{s}\right)$ to the decay of the $Z$ boson into bottom quarks, Phys. Lett. B 426 (1998) 125 [hep-ph/9712228] [INSPIRE].

[30] J.A.M. Vermaseren, New features of FORM, math-ph/0010025 [INSPIRE].

[31] M. Tentyukov and J.A.M. Vermaseren, The multithreaded version of FORM, Comput. Phys. Commun. 181 (2010) 1419 [hep-ph/0702279] [INSPIRE].

[32] M. Steinhauser, MATAD: A program package for the computation of MAssive TADpoles, Comput. Phys. Commun. 134 (2001) 335 [hep-ph/0009029] [INSPIRE].

[33] D.R.T. Jones, Two Loop Diagrams in Yang-Mills Theory, Nucl. Phys. B 75 (1974) 531 [INSPIRE].

[34] W.E. Caswell, Asymptotic Behavior of Nonabelian Gauge Theories to Two Loop Order, Phys. Rev. Lett. 33 (1974) 244 [InSPIRE].

[35] O.V. Tarasov and A.A. Vladimirov, Two Loop Renormalization of the Yang-Mills Theory in an Arbitrary Gauge, Sov. J. Nucl. Phys. 25 (1977) 585 [inSPIRE]. 